\title{
A wavenumber independent boundary element method for an acoustic scattering problem
}

Article

Published Version

Langdon, S. and Chandler-Wilde, S. N. (2006) A wavenumber independent boundary element method for an acoustic scattering problem. SIAM Journal on Numerical Analysis (SINUM), 43 (6). pp. 2450-2477. ISSN 0036-1429 Available at https://centaur.reading.ac.uk/4902/

It is advisable to refer to the publisher's version if you intend to cite from the work. See Guidance on citing.

Publisher: Society for Industrial and Applied Mathematics

Publisher statement: Copyright ( 2006 , Society for Industrial and Applied Mathematics

All outputs in CentAUR are protected by Intellectual Property Rights law, including copyright law. Copyright and IPR is retained by the creators or other copyright holders. Terms and conditions for use of this material are defined in the End User Agreement.

www.reading.ac.uk/centaur

\section{CentAUR}


Central Archive at the University of Reading

Reading's research outputs online 


\title{
A WAVENUMBER INDEPENDENT BOUNDARY ELEMENT METHOD FOR AN ACOUSTIC SCATTERING PROBLEM*
}

\author{
S. LANGDON ${ }^{\dagger}$ AND S. N. CHANDLER-WILDE ${ }^{\dagger}$
}

\begin{abstract}
In this paper we consider the impedance boundary value problem for the Helmholtz equation in a half-plane with piecewise constant boundary data, a problem which models, for example, outdoor sound propagation over inhomogeneous flat terrain. To achieve good approximation at high frequencies with a relatively low number of degrees of freedom, we propose a novel Galerkin boundary element method, using a graded mesh with smaller elements adjacent to discontinuities in impedance and a special set of basis functions so that, on each element, the approximation space contains polynomials (of degree $\nu$ ) multiplied by traces of plane waves on the boundary. We prove stability and convergence and show that the error in computing the total acoustic field is $\mathcal{O}\left(N^{-(\nu+1)} \log ^{1 / 2} N\right)$, where the number of degrees of freedom is proportional to $N \log N$. This error estimate is independent of the wavenumber, and thus the number of degrees of freedom required to achieve a prescribed level of accuracy does not increase as the wavenumber tends to infinity.
\end{abstract}

Key words. Galerkin method, high frequency, Helmholtz equation

AMS subject classifications. 35J05, 65R20

DOI. $10.1137 /$ S0036142903431936

1. Introduction. High-frequency scattering problems are of enormous interest to the mathematics, physics, and engineering communities, with applications to electromagnetic scattering, radar problems, high frequency acoustics, and geophysical waves. Although these problems have a long pedigree, their numerical solution continues to pose considerable difficulties. Many problems of scattering of time-harmonic acoustic or electromagnetic waves can be formulated as the Helmholtz equation

$$
\Delta u+k^{2} u=0,
$$

in $\mathbb{R}^{d} \backslash \Omega, d=2,3$, supplemented with appropriate boundary conditions. Here $\Omega$ is the scattering object and $k>0$ (the wavenumber) is an arbitrary positive constant, proportional to the frequency of the incident wave.

Standard schemes for solving (1.1) become prohibitively expensive as $k \rightarrow \infty$. For standard boundary element or finite element schemes, where the approximation space typically consists of piecewise polynomials, the number of degrees of freedom per wavelength must remain fixed in order to maintain accuracy, with the rule of thumb in the engineering literature a requirement for 6 to 10 elements per wavelength. Often in applications this results in excessively large systems when the wavelength is small compared to the size of the obstacle. These difficulties have been well documented; see, for example, [44, 45]. For the finite element method the situation is arguably worse in that additional pollution effects are known to be important [5, 33], these being phase errors in wave propagation across the domain, so that the degrees of freedom per wavelength need to increase somewhat to retain accuracy as $k$ increases.

The development of more efficient numerical schemes for high frequency scattering problems has attracted much recent attention in the literature. In the case of

\footnotetext{
${ }^{*}$ Received by the editors July 22, 2003; accepted for publication (in revised form) July 14, 2005; published electronically January 6, 2006. This work was supported by the EPSRC via grant GR/M59433/01 and by a Leverhulme Trust Early Career Fellowship awarded to the first author.

http://www.siam.org/journals/sinum/43-6/43193.html

${ }^{\dagger}$ Department of Mathematics, University of Reading, Whiteknights, PO Box 220, Berkshire RG6 6AX, UK (s.langdon@reading.ac.uk, s.n.chandler-wilde@reading.ac.uk).
} 
boundary element methods, a great deal of effort has focused on the fast solution of the large systems which arise, using preconditioned iterative methods (e.g., [22]) combined with fast multipole (e.g., $[24,25]$ ) or fast Fourier transform based methods (e.g., $[9,21])$ to carry out the matrix-vector multiplications efficiently. The reduction in the computing cost achieved by the use of these schemes increases the upper limit on the frequency for which accurate results can be obtained in a reasonable time. However, as the size of the system still grows at least linearly with $k$ in two dimensions (2D), quadratically in three dimensions (3D), this upper limit is not removed altogether.

An increasingly popular approach in the literature for higher frequencies is to use either a finite element or a boundary element method in which the approximation space is enriched with plane wave or Bessel function solutions of (1.1), in order to represent efficiently the highly oscillatory solution when $k$ is large. This idea has been applied to both finite element (e.g., [4, 13, 41, 29, 14, 43]) and boundary element schemes $[26,1,24,44,45,20,43,27,10,46,28]$. Promising numerical results are reported, but most of the papers are lacking in mathematical analysis, especially with regard to how any error estimates depend on the wavenumber $k$. As the present paper follows the same general approach of enriching the approximation space, we survey this body of work in a little more detail.

The methods in this category fall approximately into three groups, distinguished by how the enrichment is carried out. In one group the distinguishing feature is that a large number of solutions of the Helmholtz equation are used to form the approximation space. Most commonly the approximation space consists of standard finite element basis functions multiplied by plane waves traveling in a large number of directions, approximately uniformly distributed on the unit circle (in 2D) or sphere (in 3D). This is the approach in the generalized finite element method of Babuška and Melenk [4], the ultra weak variational formulation of Cessenat and Després [13, 14], and the least squares method of Monk and Wang [41]; see also [43, 32, 37]. In the boundary element context this approach is used in the microlocal discretization method of de La Bourdonnaye et al. [26, 27] and in the work of Perrey-Debain et al. [44, 45, 43, 46]. The theoretical analysis carried out (e.g., [4]) and computational results (e.g., [43]) confirm that these methods converge very rapidly as the number of plane wave directions used increases. Moreover, the computational results suggest that to achieve a required accuracy, the number of degrees of freedom needed is reduced by a large factor compared with conventional $h$-version finite or boundary element methods. However, in the case of boundary element methods, while constants of proportionality are reduced very significantly [44, 45, 43, 46], it is not clear that, asymptotically, the number of degrees of freedom increases any less fast than linearly with $k$ for $2 \mathrm{D}$ problems, the same rate of increase as for conventional boundary element methods.

At the other extreme, the second group of papers, using direct integral equation methods, are distinguished by using only one solution of the Helmholtz equation to enrich the approximation space, namely, the known incident field. This approach amounts to applying conventional boundary element methods to the ratio of the total field to the incident field, rather than to the total field directly. This simple idea, employed for the impedance boundary value problem we consider in this paper in [15], seems particularly appropriate in the case of smooth convex obstacles, as physical optics predicts that this ratio is approximately constant on the illuminated side and approximately zero on the shadow side at high frequencies. This approach is 
used for smooth convex obstacles in 2D in [1], where a standard Galerkin boundary element method with uniform mesh is applied to the ratio of the scattered field to the incident field. In fact, this paper appears to be the first in which the dependence of the error estimates on the wavenumber $k$ is indicated. The error estimate stated in [1] is that the relative error in the best approximation from a boundary element space of piecewise polynomials of degree $\leq \nu$ is $\mathcal{O}\left(h^{\nu}\right)+\mathcal{O}\left(\left(h k^{1 / 3}\right)^{\nu+1}\right)$. While clearly better than the (at least) linear dependence on $k$ of conventional boundary element methods, the number of degrees of freedom needed to maintain accuracy is still predicted to grow like $k^{1 / 3}$ as $k$ increases, and moreover the analysis does not guarantee that the Galerkin method solution is close to this best approximation in the limit as $k \rightarrow \infty$.

The method of [1] is applied in [24], where results for realistic 3D scatterers are shown. This approach has also recently been applied in [10]. In this latter paper, which focuses on 2D scattering by a sound-soft circular obstacle, the numerical scheme is not completely defined. However, one of the main features of the numerical scheme is that a coordinate stretching is carried out in a $k$-dependent neighborhood of the shadow boundary (of length $O\left(k^{-1 / 3}\right)$ ). The numerical results in $[10,11]$ for scattering by a circle suggest that after this transformation, the slowly varying normal derivative of the ratio of scattered to incident field can be approximated using Fourier series basis functions in the $L^{2}$ norm with a number of degrees of freedom which remains fixed as the wavenumber $k$ tends to infinity. The authors do not attempt to establish this wavenumber independence theoretically by a rigorous error analysis.

The third group of papers is intermediate in approach between the first and second groups, attempting to identify, by geometrical optics or geometrical theory of diffraction considerations or otherwise, the important wave propagation directions at high frequency. They then incorporate the oscillatory part of this high-frequency asymptotics into the approximation space for the numerical solution. This is the approach in the finite element method of Giladi and Keller [29] and in the boundary integral equation method of Bruno, Geuzaine, and Reitich [12], our own recent work [20], and the present paper. The paper [12], generalizing [10], considers specifically the case of multiple scattering between two 2D convex obstacles and employs a Neumann series approach, solving for each of the multiple scatters in turn, and factoring out a geometric optics estimate of the main oscillatory behavior at each step. We remark that the distinction between the second and third groups of papers is somewhat blurred in that, arguably, for a smooth convex obstacle the only important wave direction to include in the ansatz for the scattered field and its normal derivative on the boundary is the incident wave direction.

The last two groups of papers have in common that, while the number of degrees of freedom may be reduced, very significantly, by incorporating the oscillatory behavior of the solution, the work required to compute a typical matrix entry of the linear system to be solved increases significantly. In particular, in boundary integral equation based methods, a typical entry of the full system matrix corresponds to an integration over a part of the boundary which is large in diameter compared to the wavelength so that the integrand is highly oscillatory. The problem of efficient evaluation of these integrals is tackled by the fast multipole method in [24]. In [10], ideas from the method of stationary phase are used to reduce the support of the integrand, and quadrature rules based on the trapezoidal rule, exponentially accurate for smooth periodic functions, are employed. Numerical results using this approach for 2D scattering by a circle are encouraging and appear to indicate a fixed computational cost as 
$k$ tends to infinity. A somewhat similar approach for evaluating the integrals to that of Bruno et al. [10] is employed for scattering by smooth 3D convex scatterers in [28]. We note that there has been considerable recent interest in the efficient evaluation of highly oscillatory integrals for a variety of applications; see $[34,35]$ and the references therein.

As an instance of the third group of papers, the authors and Ritter recently proposed [20] a new high-frequency boundary element method for the specific problem of $2 \mathrm{D}$ acoustic scattering by an inhomogeneous impedance plane. For this new scheme it was shown [20] that the number of degrees of freedom needed to maintain accuracy as $k \rightarrow \infty$ grows only logarithmically with $k$. This appears to be the best theoretical estimate to date for a numerical method for a scattering problem in terms of the dependence on the wavenumber.

In this paper we will be concerned with the numerical solution of the same problem, proposing modifications of the numerical scheme of [20]. For our modified scheme we are able to show, employing somewhat more elaborate arguments than those of [20], that for a fixed number of degrees of freedom the error is bounded independently of the wavenumber $k$. To our knowledge, this is the first such numerical analysis result for any scattering problem.

The problem we will consider is one of acoustic scattering of an incident wave by a planar surface with spatially varying acoustical surface impedance. This problem has attracted much attention in the literature (see, for example, [17, 30, 31, 16, 21, 6, 48]), both in its own right and also as a model of the scattering of an incident acoustic or electromagnetic wave by an infinite rough surface $[8,47,36,7]$. In the case in which there is no variation in the acoustical properties of the surface or the incident field in some fixed direction parallel to the surface, the problem is effectively twodimensional. Adopting Cartesian coordinates $0 x_{1} x_{2} x_{3}$, let this direction be that of the $x_{3}$-axis and the surface be the plane $x_{2}=0$. Assuming further that the incident wave and scattered fields are time harmonic, the total acoustic field $u^{t} \in \mathcal{C}(\bar{U}) \cap \mathcal{C}^{2}(U)$ then satisfies (1.1) in $U:=\left\{\left(x_{1}, x_{2}\right) \in \mathbb{R}^{2}: x_{2}>0\right\}$, supplemented with the impedance boundary condition

$$
\frac{\partial u^{t}}{\partial x_{2}}+\mathrm{i} k \beta u^{t}=f \quad \text { on } \Gamma:=\left\{\left(x_{1}, 0\right): x_{1} \in \mathbb{R}\right\}
$$

with $f \equiv 0$, where $k=\omega / c>0$. Here $\omega=2 \pi \mu, \mu$ is the frequency of the incident wave and $c$ is the speed of sound in $U$. The acoustic pressure at time $t$, position $\left(x_{1}, x_{2}, x_{3}\right)$, is then given by $\operatorname{Re}\left(\mathrm{e}^{-\mathrm{i} \omega t} u^{t}(x)\right)$ for $x=\left(x_{1}, x_{2}\right) \in \bar{U}$.

In outdoor sound propagation, the relative surface admittance $\beta$ depends on the frequency and the ground properties and is often assumed in modeling to be piecewise constant and constant outside some finite interval $[a, b]$ (see, for example, $[17,30,31]$ ), with $\beta$ taking a different value for each ground surface type (grassland, forest floor, road pavement, etc. [3]). Thus, for some real numbers $a=t_{0}<t_{1}<\cdots<t_{n}=b$, the relative surface admittance at $\left(x_{1}, 0\right)$ on $\Gamma$ is given by

$$
\beta\left(x_{1}\right)= \begin{cases}\beta_{j}, & x_{1} \in\left(t_{j-1}, t_{j}\right] \\ \beta_{c}, & x_{1} \in \mathbb{R} \backslash\left(t_{0}, t_{n}\right] .\end{cases}
$$

If the ground surface is to absorb rather than emit energy, the condition $\operatorname{Re} \beta \geq 0$ must be satisfied. We assume throughout that, for some $\epsilon>0$,

$$
\operatorname{Re} \beta_{c} \geq \epsilon, \quad \operatorname{Re} \beta_{j} \geq \epsilon, \quad\left|\beta_{c}\right| \leq \epsilon^{-1}, \quad\left|\beta_{j}\right| \leq \epsilon^{-1}, \quad j=1, \ldots, n .
$$



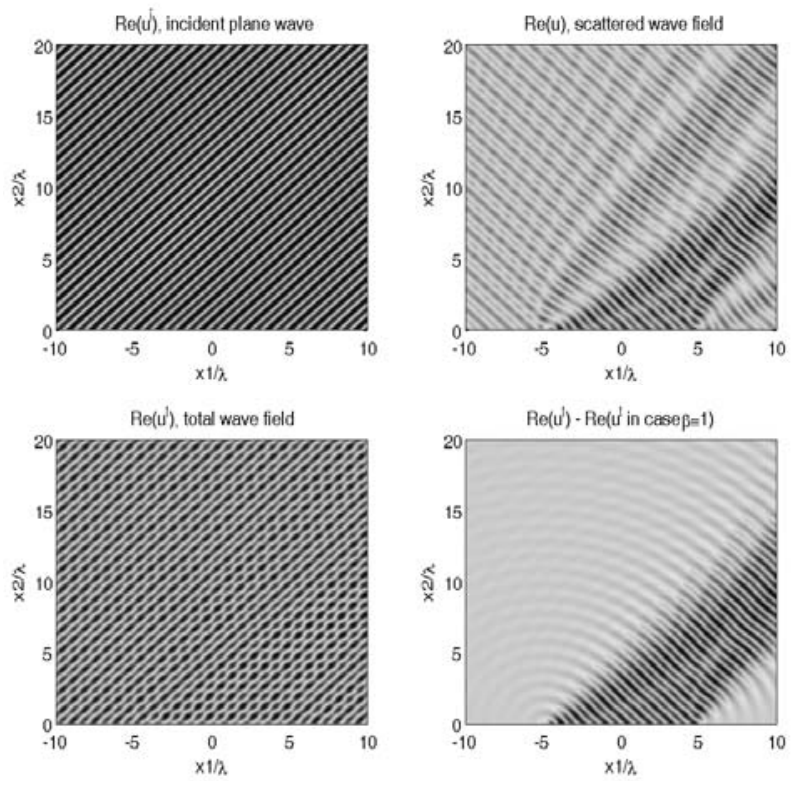

FIG. 1.1. Acoustic scattering by an impedance boundary.

For simplicity of exposition, we restrict our attention to the case of plane wave incidence, so that the incident field $u^{i}$ is given by $u^{i}(x)=\exp \left[\mathrm{i} k\left(x_{1} \sin \theta-x_{2} \cos \theta\right)\right]$, where $\theta \in(-\pi / 2, \pi / 2)$ is the angle of incidence. The reflected or scattered part of the wave field is $u:=u^{t}-u^{i} \in C(\bar{U}) \cap C^{2}(U)$, and this also satisfies (1.1) and (1.2) with

$$
f\left(x_{1}\right):=\mathrm{i} k \mathrm{e}^{\mathrm{i} k x_{1} \sin \theta}\left(\cos \theta-\beta\left(x_{1}\right)\right), \quad x_{1} \in \mathbb{R} .
$$

In Figure 1.1 we show scattering by a typical impedance plane. In this particular example, the surface admittance $\beta$ is given by

$$
\beta\left(x_{1}\right)= \begin{cases}0.505-0.3 \mathrm{i}, & x_{1} \in(-5 \lambda, 5 \lambda] \\ 1, & x_{1} \in \mathbb{R} \backslash(-5 \lambda, 5 \lambda],\end{cases}
$$

where $\lambda=c / \mu=2 \pi / k$ is the wavelength. There are discontinuities in impedance at $x_{1}=-5 \lambda$ and at $x_{1}=5 \lambda$. The incident plane wave $(\theta=\pi / 4$ in this example) can be seen in the top left and the scattered wave in the top right of Figure 1.1. This scattered wave is a combination of reflected and diffracted rays. The diffracted rays, propagating radially from the points $(-5 \lambda, 0)$ and $(5 \lambda, 0)$, can be seen more clearly in the bottom right of Figure 1.1, where we have subtracted from the total field $u^{t}$ the (known) total field in the case that $\beta \equiv 1$.

To achieve good approximations with a relatively low number of degrees of freedom, a boundary element method approach was used in [20] with ideas in the spirit of the geometrical theory of diffraction (GTD) being used to identify and subtract off the leading order behavior (namely, the incident and reflected rays) as $k \rightarrow \infty$. The remaining scattered wave (consisting of the rays diffracted at impedance discontinuities as visible in the lower right corner of Figure 1.1) can then be expressed (on the boundary $\Gamma$ ) as the product of the known oscillatory functions $\mathrm{e}^{ \pm \mathrm{i} k x_{1}}$ and unknown nonoscillatory functions denoted as $f_{j}^{ \pm}$. Rigorous bounds were established in [20] on 
the derivatives of the nonoscillatory functions $f_{j}^{ \pm}$both adjacent to and away from discontinuities in impedance. Using these bounds a Galerkin method was developed, using a graded mesh with elements very large compared to the wavelength away from discontinuities in $\beta$, in order to take advantage of the smooth behavior of $f_{j}^{ \pm}$away from these points, and a special set of basis functions so that on each element the approximation space consists of polynomials (of degree $\nu$ ) multiplied by $\mathrm{e}^{ \pm \mathrm{i} k x_{1}}$, so as to obtain a piecewise polynomial representation of the nonoscillatory functions $f_{j}^{ \pm}$. Using this approach, it was shown in [20] that the error in computing an approximation to $\left.u^{t}\right|_{\Gamma}$ on $[a, b]$ in the $L_{2}$ norm is $\mathcal{O}\left(\log ^{\nu+3 / 2}(k(b-a)) M^{-(\nu+1)}\right)$, where $M$ is the number of degrees of freedom.

In this paper we consider the same problem as in [20] and use a similar approach. We again subtract off the leading order behavior as $k \rightarrow \infty$ on each interval and express the scattered wave as a product of oscillatory and nonoscillatory functions. However, here (in section 2) we prove sharper bounds on the nonoscillatory functions $f_{j}^{ \pm}$away from impedance discontinuities. Based on these bounds, in section 3 we propose a Galerkin method similar to that in [20], but with a different approximation space. As in [20] this consists of polynomials (of degree $\nu$ ) multiplied by $\mathrm{e}^{ \pm \mathrm{i} k x_{1}}$, but unlike in [20] the choice of whether to use $\mathrm{e}^{+\mathrm{i} k x_{1}}$ or $\mathrm{e}^{-\mathrm{i} k x_{1}}$ on each element is dictated by how close the element is to each impedance discontinuity, and the graded mesh is chosen differently so that when $k$ is large compared to $N$ we do not discretize the entire domain. This is key to achieving a convergence rate independent of the wavenumber.

In section 3 we present an error analysis for this new approach, and we show that the error in computing an approximation to $\left.u^{t}\right|_{\Gamma}$ on $[a, b]$ is $\mathcal{O}\left(N^{-(\nu+1)} \log ^{1 / 2}\right.$ $\min (N, k(b-a)))$, in the $L_{2}$ norm, using a number of degrees of freedom proportional to $N \log \min (N, k(b-a))$. As $\min (N, k(b-a)) \leq N$, this error estimate shows that the error is bounded independently of $k$ for a fixed number of degrees of freedom. We believe this to be the first proof for any scattering problem that, for a fixed discretization, the error does not grow as the size (in terms of number of wavelengths) of the scattering object to be discretized tends to infinity. Moreover, for fixed $k$, as $N \rightarrow \infty$ the extra logarithmic dependence on $N$ of the error estimate and the number of degrees of freedom disappears, and we retain the same asymptotic convergence rate as in [20].

Whereas in [20] results were proved regarding only the approximation of $\left.u^{t}\right|_{\Gamma}$, here we also show, in Theorem 3.6, that the total acoustic field at any point $x \in U$ can be computed to a similar order of accuracy. In section 4 we discuss the practical implementation of our approach, and we present some numerical results demonstrating that the theoretically predicted behavior is achieved. Finally in section 5 we present some conclusions and discuss possible future extensions of the ideas presented here.

2. Integral equation formulation and regularity of the solution. In the rest of this paper, $\nu$ is the degree of the polynomial approximations used in the Galerkin method described in section 3 , and $\epsilon$, in the range $0<\epsilon<1$, is the constant in the bound (1.4). Throughout $C_{\epsilon}, C_{\nu}$, and $C_{\epsilon, \nu}$ denote constants depending only on $\epsilon, \nu$, and both $\epsilon$ and $\nu$, respectively, each not necessarily the same at each occurence.

We begin by stating the problem we wish to solve precisely and reformulating it as an integral equation. For $H \geq 0$, let $U_{H}:=\left\{\left(x_{1}, x_{2}\right): x_{2}>H\right\}$ and $\Gamma_{H}:=$ $\left\{\left(x_{1}, H\right): x_{1} \in \mathbb{R}\right\}$. To determine the scattered field $u$ uniquely we impose the radiation condition proposed in [16] that, for some $H>0, u$ can be written in the 
half plane $U_{H}$ as the double layer potential

$$
u(x)=\int_{\Gamma_{H}} \frac{\partial H_{0}^{(1)}(k|x-y|)}{\partial y_{2}} \phi(y) \mathrm{d} s(y), \quad x \in U_{H},
$$

for some density $\phi \in L_{\infty}\left(\Gamma_{H}\right)$, where $H_{0}^{(1)}$ is the Hankel function of the first kind of order zero. The boundary value problem that we wish to solve for $u$ is thus as follows.

Boundary value problem. Given $k>0$ (the wavenumber), $\theta \in(-\pi / 2, \pi / 2)$ (the angle of incidence) and $\beta$ given by (1.3), find $u \in \mathcal{C}(\bar{U}) \cap \mathcal{C}^{2}(U)$ such that

(i) $u$ is bounded in the horizontal strip $U \backslash U_{H}$ for every $H>0$;

(ii) $u$ satisfies the Helmholtz equation (1.1) in $U$;

(iii) $u$ satisfies the impedance boundary condition (1.2) on $\Gamma$ (in the weak sense explained in [16]), with $f \in L_{\infty}(\Gamma)$ given by (1.5);

(iv) $u$ satisfies the radiation condition (2.1), for some $H>0$ and $\phi \in L_{\infty}\left(\Gamma_{H}\right)$.

For $\beta^{*} \in \mathbb{C}$ with $\operatorname{Re} \beta^{*}>0$ let $G_{\beta^{*}}(x, y)$ denote the Green's function for the above problem in the case of constant relative surface impedance, which satisfies (1.2), with $\beta \equiv \beta^{*}$ and $f \equiv 0$, and the standard Sommerfeld radiation and boundedness conditions. Explicit representations and efficient calculation methods for $G_{\beta^{*}}$ are discussed in [18]. We shall require later the following bounds on $G_{\beta^{*}}[20,(2.9)$, (2.10)], which hold provided $\operatorname{Re} \beta^{*} \geq \epsilon$ and $\left|\beta^{*}\right| \leq \epsilon^{-1}$ :

$$
\begin{aligned}
& \left|G_{\beta^{*}}(x, y)\right| \leq \frac{C_{\epsilon}\left(1+k x_{2}\right)}{(k|x-y|)^{3 / 2}}, \quad x \in \bar{U}, y \in \Gamma, x \neq y, \\
& \left|G_{\beta^{*}}(x, y)\right| \leq C_{\epsilon}(1-\log (k|x-y|)), \quad x \in \bar{U}, y \in \Gamma, 0<k|x-y| \leq 1 .
\end{aligned}
$$

The following result is shown in [20].

THEOREM 2.1. If u satisfies the above boundary value problem, then

$$
u(x)=\int_{\Gamma} G_{\beta^{*}}(x, y)\left(\mathrm{i} k\left(\beta(y)-\beta^{*}\right) u(y)-f(y)\right) \mathrm{d} s(y), \quad x \in \bar{U} .
$$

Conversely, if $\left.u\right|_{\Gamma} \in B C(\Gamma)$ (the space of bounded and continuous functions on $\Gamma$ ) and $u$ satisfies (2.4), for some $\beta^{*}$ with $\operatorname{Re} \beta^{*}>0$, then u satisfies the above boundary value problem. Moreover, (2.4) has exactly one solution with $\left.u\right|_{\Gamma} \in B C(\Gamma)$, and hence the boundary value problem has exactly one solution.

We denote the (known) solution of the above boundary value problem in the special case $\beta \equiv \beta^{*}$ by $u_{\beta^{*}}$ and the corresponding total field by $u_{\beta^{*}}^{t}:=u^{i}+u_{\beta^{*}}$. Then it is easily seen [20] that $u_{\beta^{*}}$ is the plane wave $u_{\beta^{*}}(x)=R_{\beta^{*}}(\theta) \exp \left[\mathrm{i} k\left(x_{1} \sin \theta+x_{2} \cos \theta\right)\right]$, where $R_{\beta^{*}}(\theta):=\left(\cos \theta-\beta^{*}\right) /\left(\cos \theta+\beta^{*}\right)$ is a reflection coefficient. Moreover, it is shown rigorously in $[20]$ that $u^{t}$ satisfies

$$
u^{t}(x)=u_{\beta^{*}}^{t}(x)+\mathrm{i} k \int_{\Gamma} G_{\beta^{*}}(x, y)\left(\beta(y)-\beta^{*}\right) u^{t}(y) \mathrm{d} s(y), \quad x \in \bar{U} .
$$

We note that the approximate and numerical solution of this integral equation has been extensively studied; see, for example, [42, 30, 17, 21, 20].

To make explicit the dependence on the wavenumber $k$ in the results we obtain, it is useful to introduce new, dimensionless variables. Thus, define $\phi(s):=u^{t}((s / k, 0))$, $\psi_{\beta^{*}}(s):=u_{\beta^{*}}^{t}((s / k, 0))$, and $\kappa_{\beta^{*}}(s):=G_{\beta^{*}}((s / k, 0),(0,0)), s \in \mathbb{R}$. Then $(2.5)$ restricted to $\Gamma$ is the following second kind boundary integral equation for $\phi$ :

$$
\phi(s)=\psi_{\beta^{*}}(s)+\mathrm{i} \int_{-\infty}^{\infty} \kappa_{\beta^{*}}(s-t)\left(\beta(t / k)-\beta^{*}\right) \phi(t) \mathrm{d} t, \quad s \in \mathbb{R} .
$$


It is the main concern in the remainder of the paper to solve this equation numerically in the case when $\beta^{*}=\beta_{c}$. Clearly,

$$
\psi_{\beta^{*}}(s)=\left(1+R_{\beta^{*}}(\theta)\right) \mathrm{e}^{\mathrm{i} s \sin \theta},
$$

and it is shown in [20], using the representation for $G_{\beta^{*}}$ in [18], that

$$
\begin{aligned}
(2.8) \kappa_{\beta^{*}}(s) & =\frac{\mathrm{i}}{2} H_{0}^{(1)}(|s|)+\frac{\beta^{* 2} \mathrm{e}^{\mathrm{i}|s|}}{\pi} \int_{0}^{\infty} \frac{t^{-1 / 2} e^{-|s| t}}{(t-2 \mathrm{i})^{1 / 2}\left(t^{2}-2 \mathrm{i} t-\beta^{* 2}\right)} \mathrm{d} t+C_{\beta^{*}} e^{\mathrm{i}|s|\left(1-\hat{a}_{+}\right)} \\
(2.9) \quad & =\mathrm{e}^{\mathrm{i}|s|} \check{\kappa}_{\beta^{*}}(s), \quad s \in \mathbb{R} \backslash\{0\},
\end{aligned}
$$

where $\hat{a}_{ \pm}:=1 \mp\left(1-\beta^{* 2}\right)^{\frac{1}{2}}$, with $\operatorname{Re}\left\{\left(1-\beta^{* 2}\right)^{1 / 2}\right\} \geq 0$,

$$
C_{\beta^{*}}:= \begin{cases}\frac{\beta^{*}}{\left(1-\beta^{* 2}\right)^{1 / 2}}, & \operatorname{Im} \beta^{*}<0, \operatorname{Re}\left(\hat{a}_{+}\right)<0, \\ \frac{\beta^{*}}{2\left(1-\beta^{* 2}\right)^{1 / 2}}, & \operatorname{Im} \beta^{*}<0, \operatorname{Re}\left(\hat{a}_{+}\right)=0, \\ 0, & \text { otherwise, }\end{cases}
$$

and

$$
\check{\kappa}_{\beta^{*}}(s):=\frac{1}{\pi} \int_{0}^{\infty} \frac{r^{\frac{1}{2}}(r-2 \mathrm{i})^{\frac{1}{2}}}{r^{2}-2 \mathrm{i} r-\beta^{* 2}} \mathrm{e}^{-r|s|} \mathrm{d} r+C_{\beta^{*}} e^{-\mathrm{i}|s| \hat{a}_{+}}, \quad s \in \mathbb{R} \backslash\{0\} .
$$

Clearly the only dependence on $k$ in the known terms in (2.6) is in the impedance function $\beta(t / k)$. We shall see shortly that the oscillating part of $\kappa_{\beta^{*}}(s)$ is contained in the factor $\mathrm{e}^{\mathrm{i}|s|}$ in $(2.9), \check{\kappa}_{\beta^{*}}(s)$ becoming increasingly smooth as $s \rightarrow \pm \infty$.

In view of (1.3), if we set $\beta^{*}=\beta_{c}$ in (2.6), the interval of integration reduces to the finite interval $[\tilde{a}, \tilde{b}]$, where $\tilde{a}:=k a=k t_{0}, \tilde{b}:=k b=k t_{n}$. Explicitly, (2.6) becomes

$$
\phi(s)=\psi_{\beta_{c}}(s)+\mathrm{i} \int_{\tilde{a}}^{\tilde{b}} \kappa_{\beta_{c}}(s-t)\left(\beta(t / k)-\beta_{c}\right) \phi(t) \mathrm{d} t, \quad s \in \mathbb{R},
$$

with $\psi_{\beta_{c}}$ and $\kappa_{\beta_{c}}$ given by (2.7) and (2.8), respectively, with $\beta^{*}=\beta_{c}$. This integral equation is studied, in the case $\beta_{c}=1$, in [16]. From [16, Theorem 4.17] it follows that

$$
\|\phi\|_{\infty} \leq C_{\epsilon}\left\|\psi_{1}\right\|_{\infty}=C_{\epsilon}\left|1+R_{1}(\theta)\right| \leq C_{\epsilon} \cos \theta .
$$

As in [20], and as discussed in the introduction, our numerical scheme for solving (2.11) is based on a consideration of the contribution of the reflected and diffracted ray paths in the spirit of the GTD. In particular, to leading order as $k \rightarrow \infty$, on the interval $\left(t_{j-1}, t_{j}\right)$ it seems reasonable to suppose that the total field $\phi \approx \psi_{\beta_{j}}$, the total field there would be if the whole boundary had the admittance $\beta_{j}$ of the interval $\left(t_{j-1}, t_{j}\right)$, given explicitly by $(2.7)$ with $\beta^{*}=\beta_{j}$. In fact, for $s \neq \tilde{t}_{j}:=k t_{j}$, $j=0, \ldots, n$, it follows from theorem 2.3 below that $\phi(s) \rightarrow \Psi(s)$ as $k \rightarrow \infty$, where

$$
\Psi(s):= \begin{cases}\psi_{\beta_{j}}(s), \quad s \in\left(\tilde{t}_{j-1}, \tilde{t}_{j}\right], \quad j=1, \ldots, n, \\ \psi_{\beta_{c}}(s), \quad s \in \mathbb{R} \backslash\left(\tilde{t}_{0}, \tilde{t}_{n}\right] .\end{cases}
$$

In our numerical scheme we compute the difference between $\phi$ and $\Psi$, i.e.,

$$
\Phi(s):=\phi(s)-\Psi(s), \quad s \in \mathbb{R},
$$


which may be thought of as the correction to the leading order field due to scattering from impedance discontinuities. Clearly, from (2.11) we have that

$$
\Phi=\Psi_{\beta}^{\beta_{c}}+K_{\beta}^{\beta_{c}} \Phi,
$$

where $\Psi_{\beta}^{\beta_{c}} \in L_{\infty}(\mathbb{R})$ is given by $\Psi_{\beta}^{\beta_{c}}:=\psi_{\beta_{c}}-\Psi+K_{\beta}^{\beta_{c}} \Psi$, and

$$
K_{\beta}^{\beta_{c}} \chi(s):=\mathrm{i} \int_{\tilde{a}}^{\tilde{b}} \kappa_{\beta_{c}}(s-t)\left(\beta(t / k)-\beta_{c}\right) \chi(t) \mathrm{d} t .
$$

Equation (2.15) will be the integral equation that we solve numerically. By setting $\beta^{*}=\beta_{j}$ in (2.6) we obtain explicit expressions for $\Phi$ on each subinterval, namely,

$$
\Phi(s)=\mathrm{e}^{\mathrm{i} s} f_{j}^{+}\left(s-\tilde{t}_{j-1}\right)+\mathrm{e}^{-\mathrm{i} s} f_{j}^{-}\left(\tilde{t}_{j}-s\right), \quad s \in\left(\tilde{t}_{j-1}, \tilde{t}_{j}\right], j=1, \ldots, n,
$$

where for $j=1, \ldots, n, f_{j}^{+}, f_{j}^{-} \in \mathcal{C}[0, \infty)$ are defined by

$$
\begin{aligned}
& f_{j}^{+}(r):=\int_{-\infty}^{\tilde{t}_{j-1}} \check{\kappa}_{\beta_{j}}\left(r+\tilde{t}_{j-1}-t\right) \mathrm{e}^{-\mathrm{i} t} \mathrm{i}\left(\beta(t / k)-\beta_{j}\right) \phi(t) \mathrm{d} t, \\
& f_{j}^{-}(r):=\int_{\tilde{t}_{j}}^{\infty} \check{\kappa}_{\beta_{j}}\left(t-\tilde{t}_{j}+r\right) \mathrm{e}^{\mathrm{i} t} \mathrm{i}\left(\beta(t / k)-\beta_{j}\right) \phi(t) \mathrm{d} t
\end{aligned}
$$

with $\check{\kappa}_{\beta_{j}}$ given by (2.10) with $\beta^{*}=\beta_{j}$. Similarly, from (2.11),

$$
\Phi(s)= \begin{cases}\mathrm{e}^{\mathrm{i} s} f_{n+1}^{+}\left(s-\tilde{t}_{n}\right), & s>\tilde{t}_{n}, \\ \mathrm{e}^{-\mathrm{i} s} f_{0}^{-}\left(\tilde{t}_{0}-s\right), & s<\tilde{t}_{0},\end{cases}
$$

where $f_{n+1}^{+}, f_{0}^{-}$are given by (2.17), (2.18), respectively, with $\beta_{0}:=\beta_{c}$ and $\beta_{n+1}:=\beta_{c}$.

The first term in (2.16) can be viewed as an explicit summation of all the diffracted rays scattered at the discontinuity in impedance at $t_{j-1}$ which travel from left to right along $\left(t_{j-1}, t_{j}\right)$. Similarly, the other term in (2.16) is the contribution to the diffracted field diffracted by the discontinuity at $t_{j}$. In the remainder of this section, so as to design an efficient discretisation for $\Phi$, we investigate in detail the behavior of the integrals $f_{j}^{ \pm}$. As a first step, we prove the following bounds on $\left|\check{\kappa}_{\beta^{*}}^{(m)}(s)\right|$, for $m=0,1, \ldots, s \in(0, \infty)$, which were stated without proof in [20].

Lemma 2.2. Suppose that $\operatorname{Re} \beta^{*} \geq \epsilon,\left|\beta^{*}\right| \leq \epsilon^{-1}$ hold for some $\epsilon>0$. Then, for $m=0,1, \ldots$, there exist constants $c_{m}$, dependent only on $m$ and $\epsilon$, such that

$$
\begin{aligned}
& \left|\check{\kappa}_{\beta^{*}}^{(m)}(s)\right| \leq\left\{\begin{array}{ll}
c_{m}(1+|\log s|), & m=0, \\
c_{m} s^{-m}, & m \geq 1,
\end{array} \quad \text { for } 0<s \leq 1,\right. \\
& \left|\check{\kappa}_{\beta^{*}}^{(m)}(s)\right| \leq c_{m} s^{-\frac{3}{2}-m} \quad \text { for } s>1 .
\end{aligned}
$$

Proof. Throughout the proof, $c_{m}$ is a constant dependent only on $m$ and $\epsilon$, not necessarily the same at each occurrence. Let

$$
F(z):=\frac{z^{1 / 2}(z-2 \mathrm{i})^{1 / 2}}{z^{2}-2 \mathrm{i} z-\beta^{*^{2}}}=\frac{z^{1 / 2}(z-2 \mathrm{i})^{1 / 2}}{\left(z-\mathrm{i} \hat{a}_{+}\right)\left(z-\mathrm{i} \hat{a}_{-}\right)}, \quad z \in \mathbb{C},
$$

where $\operatorname{Re} z^{1 / 2}, \operatorname{Re}(z-2 \mathrm{i})^{1 / 2} \geq 0$, and $\hat{a}_{ \pm}=1 \mp \sqrt{1-\beta^{* 2}}$, as before, with $\operatorname{Re} \sqrt{1-\beta^{*^{2}}}$ $\geq 0$. Then $F(z)$ has simple poles at $z=\mathrm{i} \hat{a}_{+}$(which may lie near the real axis if Re $\hat{a}_{+}$is 
small) and $z=\mathrm{i} \hat{a}_{-}$(which cannot lie near the real axis as Re $\hat{a}_{-} \geq 1$ ). Recalling (2.10) we then have, at least provided $\operatorname{Re} \hat{a}_{+} \neq 0$ or $\operatorname{Im} \hat{a}_{+}>0$, so that the pole at $\mathrm{i} \hat{a}_{+}$does not lie on the positive real axis,

$$
\left|\check{\kappa}_{\beta^{*}}^{(m)}(s)\right| \leq \frac{1}{\pi}\left|\int_{0}^{\infty} F(r) r^{m} \mathrm{e}^{-r s} \mathrm{~d} r\right|+\left|C_{\beta^{*}} \hat{a}_{+}^{m} \mathrm{e}^{\operatorname{Im} \hat{a}_{+} s}\right|, \quad s>0 .
$$

Now, since $\operatorname{Re} \beta^{*} \geq \epsilon$, it is easy to see that $\operatorname{Im} \hat{a}_{+}=0$ if and only if $\beta^{*} \in[\epsilon, 1]$, and in this case $\operatorname{Re} \hat{a}_{+} \geq 1-\sqrt{1-\epsilon^{2}}=\epsilon^{2} /\left(1+\sqrt{1-\epsilon^{2}}\right)>\epsilon^{2} / 2$. We thus define $S_{\epsilon}:=\left\{\beta^{*}: \operatorname{Re} \beta^{*} \geq \epsilon,\left|\beta^{*}\right| \leq \epsilon^{-1}, \operatorname{Re} \hat{a}_{+} \leq \epsilon^{2} / 4\right\}$. Then $S_{\epsilon}$ is closed and bounded, and $\left|\operatorname{Im} \hat{a}_{+}\right|$and $\left|\sqrt{1-\beta^{* 2}}\right|$ are both continuous and nonzero on $S_{\epsilon}$. Thus, for some $\eta>0$,

$$
\left|\operatorname{Im} \hat{a}_{+}\right| \geq \eta \quad \text { and } \quad\left|\sqrt{1-\beta^{* 2}}\right| \geq \eta
$$

for all $\beta^{*} \in S_{\epsilon}$.

Next, we note that if $\operatorname{Re} \hat{a}_{+}>0$, then $C_{\beta^{*}}=0$, while if $\operatorname{Re} \hat{a}_{+} \leq 0$, then $\beta^{*} \in S_{\epsilon}$, so that $(2.22)$ holds. Moreover, if $C_{\beta^{*}} \neq 0$, then $\operatorname{Im} \beta^{*}<0$, and so $\operatorname{Im} \hat{a}_{+}<0$. Since also $\left|\hat{a}_{ \pm}\right| \leq 1+\sqrt{1+\epsilon^{-2}}$, we see that

$$
\left|C_{\beta^{*}} \hat{a}_{+}^{m} \mathrm{e}^{\operatorname{Im} \hat{a}_{+} s}\right| \leq c_{m} \mathrm{e}^{-\eta s}, \quad s>0 .
$$

We turn to bounding the first term on the right-hand side of (2.21). To do this we consider the two cases $\left|\operatorname{Re} \hat{a}_{+}\right|>\epsilon^{2} / 4$ and $\left|\operatorname{Re} \hat{a}_{+}\right| \leq \epsilon^{2} / 4$ separately.

First, suppose $\left|\operatorname{Re} \hat{a}_{+}\right|>\epsilon^{2} / 4$. Then

$$
|F(r)| \leq C_{\epsilon} r^{1 / 2}, \quad r>0,
$$

and thus

$$
\left|\int_{0}^{\infty} F(r) r^{m} \mathrm{e}^{-r s} \mathrm{~d} r\right| \leq C_{\epsilon} \int_{0}^{\infty} r^{m+1 / 2} \mathrm{e}^{-r s} \mathrm{~d} r \leq c_{m} s^{-m-3 / 2}, \quad s>0 .
$$

This bound suffices when $s>1$, but for $0<s \leq 1$ we need a sharper bound.

We proceed by establishing bounds on the $m$ th derivatives of the first two terms on the right-hand side of $(2.8)$ for $0<s \leq 1$. It can easily be deduced from the power series representations defining the Bessel functions that there exist constants $C_{j}, j=0, \ldots$, such that, for $0<z \leq 1$,

$$
\begin{aligned}
\left|H_{0}^{(1)}(z)\right| & \leq C_{0}(1+|\log z|), \\
\left|\frac{d^{m}}{d z^{m}} H_{0}^{(1)}(z)\right| & \leq C_{m} z^{-m}, \quad m=1,2, \ldots .
\end{aligned}
$$

Next note that, for $0<s \leq 1$, the $m$ th derivative of the second term in (2.8) has absolute value not more than

$$
\begin{aligned}
\left|\frac{\beta^{* 2}}{\pi} \int_{0}^{\infty} \frac{(\mathrm{i}-t)^{m} \mathrm{e}^{-s t} t^{-1 / 2} \mathrm{~d} t}{(t-2 \mathrm{i})^{1 / 2}\left(t^{2}-2 \mathrm{i} t-\beta^{* 2}\right)}\right| & \leq \frac{\epsilon^{-2}}{\pi} \int_{0}^{\infty} \frac{\left(1+t^{2}\right)^{m / 2} \mathrm{e}^{-s t} t^{-1 / 2} \mathrm{~d} t}{\left(t^{2}+4\right)^{1 / 4}\left|\left(t-\mathrm{i} \hat{a}_{+}\right)\left(t-\mathrm{i} \hat{a}_{-}\right)\right|} \\
& \leq C_{\epsilon}\left[\int_{0}^{1} t^{-1 / 2} \mathrm{~d} t+\int_{1}^{\infty} t^{m-1} \mathrm{e}^{-s t} \mathrm{~d} t\right] \\
& \leq \begin{cases}C_{\epsilon}(1-\log s), & m=0, \\
c_{m} s^{-m}, & m=1,2, \ldots\end{cases}
\end{aligned}
$$


Combining (2.25), (2.26), and (2.29) and recalling (2.9) the result follows.

Now we consider the case $0 \leq \operatorname{Re} \hat{a}_{+} \leq \epsilon^{2} / 4$. (The proof for the case $-\epsilon^{2} / 4 \leq$ Re $\hat{a}_{+}<0$ is similar.) As $\beta \in S_{\epsilon},(2.22)$ holds. If $\operatorname{Im} \hat{a}_{+}>0$, then the bounds (2.23) and (2.28) hold and we proceed as above. If $\operatorname{Im} \hat{a}_{+}<0$, however, $F(z)$ has a pole at $z=\mathrm{i} \hat{a}_{+}$with $\operatorname{Re}\left(\mathrm{i} \hat{a}_{+}\right)>\eta, 0 \leq \operatorname{Im}\left(\mathrm{i} \hat{a}_{+}\right) \leq \epsilon^{2} / 4$. To bound the integrals on the left-hand side of (2.24) and (2.27) in this case, uniformly in $\beta^{*}$, we first deform the path of integration. Define $\Gamma_{\epsilon}$ to be the semicircle, center $\left(-\operatorname{Im} \hat{a}_{+}, 0\right)$, radius $\tilde{\eta}:=\min (1 / 2, \eta)$, lying in the lower half plane. (Note that by $(2.22), \operatorname{Re} z>\eta / 2$ for $z \in \Gamma_{\epsilon}$.) Let $\gamma_{\epsilon}=\left[0,-\operatorname{Im} \hat{a}_{+}-\eta / 2\right] \cup\left[-\operatorname{Im} \hat{a}_{+}+\eta / 2, \infty\right)$. Then, by Cauchy's theorem, it follows from (2.21) that, for $\operatorname{Re} \hat{a}_{+}>0$,

$$
\left|\check{\kappa}_{\beta^{*}}^{(m)}(s)\right| \leq \frac{1}{\pi}\left|\int_{\gamma_{\epsilon}} F(r) r^{m} \mathrm{e}^{-r s} \mathrm{~d} r+\int_{\Gamma_{\epsilon}} F(r) r^{m} \mathrm{e}^{-r s} \mathrm{~d} r\right|, \quad s>0 .
$$

By continuity arguments, taking the limit Re $\hat{a}_{+} \rightarrow 0^{+}$in (2.30), equation (2.30) holds also for Re $\hat{a}_{+}=0$. For $r \in \gamma_{\epsilon}$ the bound (2.23) holds, and so the integral over $\gamma_{\epsilon}$ is bounded by the right-hand side of (2.24). Further,

$$
\left|\int_{\Gamma_{\epsilon}} F(r) r^{m} \mathrm{e}^{-r s} \mathrm{~d} r\right| \leq \frac{\pi \eta}{2} \max _{r \in \Gamma_{\epsilon}}\left|F(r) r^{m} \mathrm{e}^{-r s}\right| \leq c_{m} \mathrm{e}^{-\eta s / 2},
$$

so we obtain the required bound for $s \geq 1$. To obtain the desired bound for $0<s \leq 1$ we proceed as in the case $\left|\operatorname{Re} \hat{a}_{+}\right|>\epsilon^{2} / 4$, but deforming the path of integration as above to bound the left-hand side of (2.27).

The following result is a slight sharpening of [20, Theorem 2.6], obtained by combining the bounds in Lemma 2.2 and (2.12) with the representations (2.17) and (2.18).

Theorem 2.3. Suppose (1.4) holds for some $\epsilon>0$. Then, for $r>0, j=1, \ldots, n$, $m=0,1, \ldots$, there exist constants $c_{m}$, dependent only on $m$ and $\epsilon$, such that

$$
\left|f_{j}^{ \pm(m)}(r)\right| \leq c_{m} \cos \theta E_{m}(r)
$$

where

$$
\begin{aligned}
& E_{m}(r)=\left\{\begin{array}{ll}
1, & m=0, \\
1-\log r, & m=1, \\
r^{1-m}, & m \geq 2,
\end{array} \quad \text { for } 0<r \leq 1,\right. \\
& E_{m}(r)=r^{-\frac{1}{2}-m}
\end{aligned}
$$

Remark. Using the identical argument it can easily be shown that $\left|f_{n+1}^{+}{ }^{(m)}(r)\right|$, $\left|f_{0}^{-(m)}(r)\right| \leq c_{m} \cos \theta E_{m}(r), r>0$, for $m=0,1, \ldots$, where $c_{m}$ is the same constant as in Theorem 2.3.

To prove the main result of this section, a sharper bound on $\left|f_{j}^{ \pm}(r)\right|$ when $r>1$ (Theorem 2.6), we require the bounds in the following two lemmas.

Lemma 2.4. Suppose $p<-1$ and $q \leq 0$. Then there exists a constant $C$, independent of $r$, such that, for $r \geq 1$,

$$
\int_{0}^{\infty}(t+r)^{p}(1+t)^{q} \mathrm{~d} t \leq \begin{cases}C r^{p+q+1}, & q \neq-1 \\ C r^{p} \log (1+r), & q=-1 .\end{cases}
$$


Proof.

$$
\int_{0}^{\infty}(t+r)^{p}(1+t)^{q} \mathrm{~d} t \leq r^{p} \int_{0}^{r}(1+t)^{q} \mathrm{~d} t+r^{q} \int_{r}^{\infty}(t+r)^{p} \mathrm{~d} t
$$

and the result follows.

\section{$\square$}

Lemma 2.5. Suppose $q \leq 0$. Then there exists a constant $C$, independent of $r$ and $D$, such that, for $r \geq 1$ and $D>0$,

$$
\int_{0}^{2 D}(s+r)^{-3 / 2}(1+2 D-s)^{q} \mathrm{~d} s \leq \begin{cases}C r^{-3 / 2}, & q<-1, \\ C r^{-3 / 2} \log (1+r), & q=-1, \\ C r^{-1 / 2+q}, & -1<q \leq 0 .\end{cases}
$$

Proof. Splitting the integration range as $[0,2 D]=[0, D] \cup[D, 2 D]$, and making the change of variable $t:=2 D-s$, we see that

$$
\int_{0}^{2 D}(s+r)^{-3 / 2}(1+2 D-s)^{q} \mathrm{~d} s \leq I_{A}+I_{B}
$$

where

$$
I_{A}:=(1+D)^{q} \int_{0}^{D}(s+r)^{-3 / 2} \mathrm{~d} s, \quad I_{B}:=(D+r)^{-3 / 2} \int_{0}^{D}(1+t)^{q} \mathrm{~d} t .
$$

Further,

$$
\begin{aligned}
I_{A} & =\frac{2 D(1+D)^{q}}{r^{1 / 2}(D+r)^{1 / 2}\left((D+r)^{1 / 2}+r^{1 / 2}\right)} \\
& \leq \frac{2(1+D)^{q+1}}{r^{1 / 2}(D+r)} \leq \begin{cases}2 r^{-3 / 2}, & q \leq-1, \\
2 r^{-1 / 2+q}, & -1<q \leq 0 .\end{cases}
\end{aligned}
$$

For $q \neq-1, I_{B}=(1+q)^{-1}(D+r)^{-3 / 2}\left((1+D)^{q+1}-1\right)$. Thus

$$
|1+q| I_{B} \leq \begin{cases}r^{-3 / 2}, & q<-1 \\ r^{-1 / 2+q}, & -1<q \leq 0 .\end{cases}
$$

To bound $I_{B}$ in the case that $q=-1$ we need to consider the cases $r \geq D$ and $r<D$ separately. For $r \geq D$,

$$
I_{B}=(D+r)^{-3 / 2} \log (1+D) \leq r^{-3 / 2} \log (1+r) .
$$

For $r<D$ we split the range of integration as $[0, D]=[0, r] \cup[r, D]$ and note that

$$
\begin{gathered}
(D+r)^{-3 / 2} \int_{0}^{r}(1+t)^{-1} \mathrm{~d} t \leq r^{-3 / 2} \log (1+r), \\
(D+r)^{-3 / 2} \int_{r}^{D}(1+t)^{-1} \mathrm{~d} t \leq \frac{(D-r)}{(D+r)^{3 / 2}(1+r)} \leq r^{-3 / 2} .
\end{gathered}
$$

This completes the proof.

We are now ready to prove the main result of this section, the following sharper bound on $\left|f_{j}^{ \pm}(r)\right|$ when $r>1$, on which the design of our numerical scheme is based.

Theorem 2.6. Suppose (1.4) holds for some $\epsilon>0$. Then for $r>1, j=0, \ldots, n$,

$$
\left|f_{j+1}^{+}(r)\right|,\left|f_{j}^{-}(r)\right| \leq C_{\epsilon} \frac{r^{-3 / 2} n^{3}}{\cos \theta} .
$$


Proof. First we consider $f_{j}^{-}(r)$. Recalling $(2.14)$, for $j=0, \ldots, n, f_{j}^{-}(r)=$ $I_{1}(r)+I_{2}(r)$, where

$$
\begin{aligned}
& I_{1}(r):=\int_{\tilde{t}_{j}}^{\infty} \check{\kappa}_{\beta_{j}}\left(t-\tilde{t}_{j}+r\right) \mathrm{e}^{\mathrm{i} t} \mathrm{i}\left(\beta(t / k)-\beta_{j}\right) \Psi(t) \mathrm{d} t, \\
& I_{2}(r):=\int_{\tilde{t}_{j}}^{\infty} \check{\kappa}_{\beta_{j}}\left(t-\tilde{t}_{j}+r\right) \mathrm{e}^{\mathrm{i} t} \mathrm{i}\left(\beta(t / k)-\beta_{j}\right) \Phi(t) \mathrm{d} t .
\end{aligned}
$$

We begin by establishing a bound on $I_{1}$. Recalling (2.13) and (2.7),

$$
\begin{gathered}
I_{1}(r)=\sum_{m=j+1}^{n} \mathrm{i}\left(\beta_{m}-\beta_{j}\right) \int_{\tilde{t}_{m-1}}^{\tilde{t}_{m}} \check{\kappa}_{\beta_{j}}\left(t-\tilde{t}_{j}+r\right)\left(1+R_{\beta_{m}}(\theta)\right) \mathrm{e}^{\mathrm{i} t(\sin \theta+1)} \mathrm{d} t \\
\quad+\mathrm{i}\left(\beta_{c}-\beta_{j}\right) \int_{\tilde{t}_{n}}^{\infty} \check{\kappa}_{\beta_{j}}\left(t-\tilde{t}_{j}+r\right)\left(1+R_{\beta_{c}}(\theta)\right) \mathrm{e}^{\mathrm{i} t(\sin \theta+1)} \mathrm{d} t .
\end{gathered}
$$

Integrating by parts,

$$
\begin{aligned}
I_{1}(r)=\sum_{m=j+1}^{n} & \frac{\left(\beta_{m}-\beta_{j}\right)\left(1+R_{\beta_{m}}(\theta)\right)}{\sin \theta+1}\left(\left[\check{\kappa}_{\beta_{j}}\left(t-\tilde{t}_{j}+r\right) \mathrm{e}^{\mathrm{i} t(\sin \theta+1)}\right]_{\tilde{t}_{m-1}}^{\tilde{t}_{m}}\right. \\
& \left.-\int_{\tilde{t}_{m-1}}^{\tilde{t}_{m}} \check{\kappa}_{\beta_{j}}^{\prime}\left(t-\tilde{t}_{j}+r\right) \mathrm{e}^{\mathrm{i} t(\sin \theta+1)} \mathrm{d} t\right) \\
+ & \frac{\left(\beta_{c}-\beta_{j}\right)\left(1+R_{\beta_{c}}(\theta)\right)}{\sin \theta+1}\left(\left[\check{\kappa}_{\beta_{j}}\left(t-\tilde{t}_{j}+r\right) \mathrm{e}^{\mathrm{i} t(\sin \theta+1)}\right]_{\tilde{t}_{n}}^{\infty}\right. \\
& \left.-\int_{\tilde{t}_{n}}^{\infty} \check{\kappa}_{\beta_{j}}^{\prime}\left(t-\tilde{t}_{j}+r\right) \mathrm{e}^{\mathrm{i} t(\sin \theta+1)} \mathrm{d} t\right) .
\end{aligned}
$$

Now from Lemma 2.2, for $r>1$,

$$
\left|\check{\kappa}_{\beta_{j}}\left(\tilde{t}_{m}-\tilde{t}_{j}+r\right)\right| \leq C_{\epsilon}\left(\tilde{t}_{m}-\tilde{t}_{j}+r\right)^{-3 / 2} \leq C_{\epsilon} r^{-3 / 2}, \quad m=j, \ldots, n .
$$

Thus, noting that $\left|1+R_{\beta_{m}}(\theta)\right|=\left|2 \cos \theta /\left(\cos \theta+\beta_{m}\right)\right| \leq 2 \cos \theta / \epsilon$ and $\left|\beta_{m}-\beta_{j}\right| \leq 2 / \epsilon$, and using Lemma 2.2 again to bound $\check{\kappa}_{\beta_{j}}^{\prime}$, we have, for $r>1$,

$$
\begin{aligned}
\left|I_{1}(r)\right| & \leq C_{\epsilon} \frac{(n+1-j) \cos \theta}{\sin \theta+1}\left[r^{-3 / 2}+\int_{\tilde{t}_{j}}^{\infty}\left|\check{\kappa}_{\beta_{j}}^{\prime}\left(t-\tilde{t}_{j}+r\right)\right| \mathrm{d} t\right] \\
& \leq C_{\epsilon} \frac{r^{-3 / 2} n \cos \theta}{\sin \theta+1}
\end{aligned}
$$

We next bound $I_{2}$. Recalling (2.16) and (2.19),

$$
\left|I_{2}(r)\right| \leq \frac{2}{\epsilon}\left(J_{\infty}^{+}+\sum_{m=j+1}^{n}\left(J_{m}^{+}+J_{m}^{-}\right)\right),
$$

where

$$
\begin{aligned}
J_{m}^{+} & :=\int_{\tilde{t}_{m-1}}^{\tilde{t}_{m}}\left|\check{\kappa}_{\beta_{j}}\left(t-\tilde{t}_{j}+r\right)\right|\left|f_{m}^{+}\left(t-\tilde{t}_{m-1}\right)\right| \mathrm{d} t, \\
J_{m}^{-} & :=\int_{\tilde{t}_{m-1}}^{\tilde{t}_{m}}\left|\check{\kappa}_{\beta_{j}}\left(t-\tilde{t}_{j}+r\right)\right|\left|f_{m}^{-}\left(\tilde{t}_{m}-t\right)\right| \mathrm{d} t, \\
J_{\infty}^{+} & :=\int_{\tilde{t}_{n}}^{\infty}\left|\check{\kappa}_{\beta_{j}}\left(t-\tilde{t}_{j}+r\right)\right|\left|f_{n+1}^{+}\left(t-\tilde{t}_{n}\right)\right| \mathrm{d} t .
\end{aligned}
$$


First we bound $J_{\infty}^{+}$. Applying Lemma 2.2 and Theorem 2.3, and noting the remark after Theorem 2.3, for $r>1$,

$$
J_{\infty}^{+} \leq C_{\epsilon} \cos \theta \int_{\tilde{t}_{n}}^{\infty}\left(t-\tilde{t}_{n}+r\right)^{-3 / 2}\left(1+t-\tilde{t}_{n}\right)^{-1 / 2} \mathrm{~d} t \leq C_{\epsilon} r^{-1} \cos \theta
$$

making the change of variables $s=t-\tilde{t}_{n}$ and using Lemma 2.4 with $p=-3 / 2$ and $q=-1 / 2$. Arguing similarly, $J_{m}^{+} \leq C_{\epsilon} r^{-1} \cos \theta$. To bound $J_{m}^{-}$, again using Lemma 2.2 and Theorem 2.3 we have

$$
J_{m}^{-} \leq C_{\epsilon} \cos \theta \int_{\tilde{t}_{m-1}}^{\tilde{t}_{m}}\left(t-\tilde{t}_{m-1}+r\right)^{-3 / 2}\left(1+\tilde{t}_{m}-t\right)^{-1 / 2} \mathrm{~d} t \leq C_{\epsilon} r^{-1} \cos \theta,
$$

making the change of variables $s=t-\tilde{t}_{m-1}$ and using Lemma 2.5 with $D=\left(\tilde{t}_{m}-\right.$ $\left.\tilde{t}_{m-1}\right) / 2$ and $q=-1 / 2$. Thus, recalling (2.33), $\left|I_{2}(r)\right| \leq C_{\epsilon} r^{-1} n \cos \theta$.

So far in the argument we have shown that, for $r>1, j=0, \ldots, n$,

$$
\left|f_{j}^{-}(r)\right| \leq\left|I_{1}(r)\right|+\left|I_{2}(r)\right| \leq C_{\epsilon} r^{-1} n \cos \theta\left(\frac{r^{-1 / 2}}{1+\sin \theta}+1\right) .
$$

Proceeding in a similar way, we can show that, for $r>1, j=1, \ldots, n+1$,

$$
\left|f_{j}^{+}(r)\right| \leq C_{\epsilon} r^{-1} n \cos \theta\left(\frac{r^{-1 / 2}}{1-\sin \theta}+1\right) .
$$

Next, starting from (2.33)-(2.36), we can use (2.37) and (2.38) to establish sharper bounds on $I_{2}$ and hence a sharper bound on $f_{j}^{-}$. Using (2.38) in (2.36), we have for $r>1$ that

$$
\begin{aligned}
J_{\infty}^{+} & \leq C_{\epsilon} n \cos \theta \int_{\tilde{t}_{n}}^{\infty}\left(t-\tilde{t}_{n}+r\right)^{-3 / 2}\left(1+t-\tilde{t}_{n}\right)^{-1}\left(1+\frac{\left(1+t-\tilde{t}_{n}\right)^{-1 / 2}}{1-\sin \theta}\right) \mathrm{d} t \\
& \leq C_{\epsilon} r^{-3 / 2} n \cos \theta\left(\log (1+r)+(1-\sin \theta)^{-1}\right),
\end{aligned}
$$

making the change of variable $s=t-\tilde{t}_{n}$ and using Lemma 2.4 with $p=-3 / 2$ and $q=-1,-3 / 2$. Arguing similarly, we can show that

$$
J_{m}^{+} \leq C_{\epsilon} r^{-3 / 2} n \cos \theta\left(\log (1+r)+(1-\sin \theta)^{-1}\right), \quad m=j+1, \ldots, n,
$$

and, using (2.37) and Lemma 2.2,

$$
\begin{aligned}
J_{m}^{-} & \leq C_{\epsilon} n \cos \theta \int_{\tilde{t}_{m-1}}^{\tilde{t}_{m}}\left(t-\tilde{t}_{m-1}+r\right)^{-3 / 2}\left(1+\tilde{t}_{m}-t\right)^{-1}\left(1+\frac{\left(1+\tilde{t}_{m}-t\right)^{-1 / 2}}{1+\sin \theta}\right) \mathrm{d} t \\
& \leq C_{\epsilon} r^{-3 / 2} n \cos \theta\left(\log (1+r)+(1+\sin \theta)^{-1}\right),
\end{aligned}
$$

where again we make the change of variable $s=t-\tilde{t}_{m-1}$ and use Lemma 2.5 with $D:=\left(\tilde{t}_{m}-\tilde{t}_{m-1}\right) / 2$ and $q=-1,-3 / 2$. Combining this with (2.39) and (2.40),

$$
\left|I_{2}(r)\right| \leq C_{\epsilon} r^{-3 / 2} n^{2} \cos \theta\left(\log (1+r)+\left(1-\sin ^{2} \theta\right)^{-1}\right) \leq C_{\epsilon} \frac{r^{-3 / 2} \log (1+r) n^{2}}{\cos \theta} .
$$

Thus

$$
\left|f_{j}^{-}(r)\right| \leq\left|I_{1}\right|+\left|I_{2}\right| \leq C_{\epsilon} \frac{r^{-3 / 2} \log (1+r) n^{2}}{\cos \theta}
$$


In a similar way it can be proved that

$$
\left|f_{j}^{+}(r)\right| \leq C_{\epsilon} \frac{r^{-3 / 2} \log (1+r) n^{2}}{\cos \theta} .
$$

To obtain sharper bounds still on $f_{j}^{ \pm}$, removing the dependence on $\log r$ in (2.41), (2.42), we note that it follows from (2.41) and (2.42) that

$$
\int_{0}^{\infty}\left|f_{j}^{ \pm}(r)\right| \mathrm{d} r \leq C_{\epsilon} \frac{n^{2}}{\cos \theta} .
$$

Using this bound and the bounds in Lemma 2.2 in (2.34)-(2.36), we see that

$$
J_{m}^{ \pm} \leq C_{\epsilon} r^{-3 / 2} \int_{0}^{\infty}\left|f_{m}^{ \pm}(s)\right| \mathrm{d} s \leq C_{\epsilon} \frac{r^{-3 / 2} n^{2}}{\cos \theta},
$$

and an identical bound holds on $J_{\infty}^{+}$. Hence, recalling (2.33),

$$
\left|I_{2}(r)\right| \leq C_{\epsilon} \frac{r^{-3 / 2} n^{3}}{\cos \theta},
$$

and combining this with $(2.32)$ the desired bound on $f_{j}^{-}(r)$ follows. The desired bound on $f_{j}^{+}(r)$ follows similarly.

3. Galerkin method and error analysis. Our aim now is to design a numerical method for the solution of (2.15), supported by a full error analysis, for which the error bounds are independent of the parameter $k(b-a)$. To achieve this we will work in $L_{2}(\mathbb{R})$, and to that end we introduce the operator $Q: L_{\infty}(\mathbb{R}) \rightarrow L_{2}(\mathbb{R})$ defined by

$$
Q \chi(s):= \begin{cases}\chi(s), & s \in[\tilde{a}, \tilde{b}]=\left[\tilde{t}_{0}, \tilde{t}_{n}\right] \\ 0, & s \in \mathbb{R} \backslash[\tilde{a}, \tilde{b}] .\end{cases}
$$

Writing $\Phi^{*}:=Q \Phi$, and noting that $K_{\beta}^{\beta_{c}} \Phi=K_{\beta}^{\beta_{c}} \Phi^{*}$, it follows from (2.15) that

$$
\Phi^{*}-Q K_{\beta}^{\beta_{c}} \Phi^{*}=Q \Psi_{\beta}^{\beta_{c}},
$$

where $\Phi^{*}$ and $Q \Psi_{\beta}^{\beta_{c}}$ are both in $L_{2}(\mathbb{R})$.

Existence and boundedness of $\left(I-Q K_{\beta}^{\beta_{c}}\right)^{-1}: L_{2}(\mathbb{R}) \rightarrow L_{2}(\mathbb{R})$ are shown in [20], where it is also shown that the unique solution $\Phi^{*}=\left(I-Q K_{\beta}^{\beta_{c}}\right)^{-1} Q \Psi_{\beta}^{\beta_{c}}$ of (3.1) satisfies $\left\|\Phi^{*}\right\|_{2} \leq C_{1}\left\|Q \Psi_{\beta}^{\beta_{c}}\right\|_{2}$ with $C_{1}=\operatorname{Re} \beta_{c} /\left(\operatorname{Re} \beta_{c}-\left\|\beta-\beta_{c}\right\|_{\infty}\right)$ if

$$
\left|\beta_{j}-\beta_{c}\right|<\operatorname{Re} \beta_{c}, \quad j=1, \ldots, n,
$$

and $C_{1}$ unspecified but dependent only on $\epsilon$ and $\beta_{c}$ if (3.2) does not hold.

To approximate the solution $\Phi^{*}=Q \Phi$ of (3.1) we use a Galerkin method, similar to that in [20], but with the approximation space chosen in a different way so as to take advantage of our stronger bound on $\Phi$ (Theorem 2.6), in order to remove the dependence of the error estimates on $k(b-a)$. As in [20], on each interval $\left(\tilde{t}_{j-1}, \tilde{t}_{j}\right)$, we approximate $f_{j}^{+}\left(s-\tilde{t}_{j-1}\right)$ and $f_{j}^{-}\left(\tilde{t}_{j}-s\right)$ in $(2.16)$ by conventional piecewise polynomial approximations, rather than approximating $\Phi$ itself. This makes sense since, as quantified by Theorems 2.3 and 2.6 , the functions $f_{j}^{+}\left(s-\tilde{t}_{j-1}\right)$ and $f_{j}^{-}\left(\tilde{t}_{j}-s\right)$ are 
smooth (their higher order derivatives are small) away from $\tilde{t}_{j-1}$ and $\tilde{t}_{j}$, respectively. To approximate $f_{j}^{+}\left(s-\tilde{t}_{j-1}\right)$ and $f_{j}^{-}\left(\tilde{t}_{j}-s\right)$ we use piecewise polynomials of a fixed degree $\nu \geq 0$ on a graded mesh, the mesh grading adapted in an optimal way to the bounds on $f_{j}^{ \pm(m)}$ in Theorems 2.3 and 2.6.

To begin, we define a graded mesh on a general interval $[0, A]$, for $A>1$, with more mesh points near 0 and less near $A$. This mesh is identical to that defined in [20, Definition 3.1]; the difference here is in how we choose the value of $A$ when we apply this mesh to the discretization of each interval $\left[\tilde{t}_{j-1}, \tilde{t}_{j}\right]$. Whereas in $[20], A$ was chosen as a function of $\tilde{t}_{j}-\tilde{t}_{j-1}$ and the functions $f_{j}^{ \pm}$were approximated over the whole interval $\left[\tilde{t}_{j-1}, \tilde{t}_{j}\right]$, here we choose $A$ as a function of $N$, a positive integer, where the size of $N$ also determines the density of the mesh on $[0, A]$. A judicious choice of $A=A(N)$, as described below, allows us to discretize only a subsection of the interval $\left[\tilde{t}_{j-1}, \tilde{t}_{j}\right]$, near to $\tilde{t}_{j-1}$ and $\tilde{t}_{j}$, and to approximate $f_{j}^{ \pm}$by zero away from these points without harming the overall accuracy of our scheme. This is the key to achieving error estimates independent of $k(b-a)$.

The mesh we use also has similarities to that used in [40] for solving (1.1) in the case $k=\mathrm{i} \tau, \tau>0, \tau$ large, where a similar idea of only discretizing a subsection of the boundary as $k \rightarrow \infty$ was used to establish error bounds independent of $\tau$.

Definition 3.1. For $A>1$ and $N=2,3, \ldots$, the mesh $\Lambda_{N, A}=\left\{y_{0}, \ldots, y_{N+N_{A}}\right\}$ consists of the points $y_{i}=(i / N)^{q}, i=0, \ldots, N$, where $q=1+2 \nu / 3$, together with the points $y_{N+j}=A^{j / N_{A}}, j=1, \ldots, N_{A}$, where $N_{A}=\left\lceil N^{*}\right\rceil$, the smallest integer $\geq N^{*}$, and $N^{*}:=-\log A /[q \log (1-1 / N)]$.

The mesh $\Lambda_{N, A}$ is a composite mesh with a polynomial grading on $[0,1]$ and a geometric grading on $[1, A]$. The definition of $N_{A}$ ensures a smooth transition between the two parts of the mesh. Precisely, the definition of $N^{*}$ is such that, in the case $N_{A}=N^{*}$, it holds that $y_{N+1} / y_{N}=y_{N} / y_{N-1}$, so that $y_{N-1}$ and $y_{N}$ are points in both the polynomial and the geometric parts of the mesh. It is shown in [20] that the total number of subintervals $N+N_{A}$ of the mesh on $[0, A]$ satisfies

$$
N+N_{A}<\left(\frac{3}{2}+\frac{\log A}{q}\right) N
$$

Let $\Pi_{A, N, \nu}:=\left\{\sigma:\left.\sigma\right|_{\left[y_{j-1}, y_{j}\right]}\right.$ is a polynomial of degree $\left.\leq \nu, j=1, \ldots, N+N_{A}\right\}$, and let $P_{N}^{*}$ be the orthogonal projection operator from $L_{2}(0, A)$ to $\Pi_{A, N, \nu}$, so that setting $p=P_{N}^{*} f$ minimizes $\|f-p\|_{2,(0, A)}=\left\{\int_{0}^{A}|f(t)-p(t)|^{2} \mathrm{~d} t\right\}^{1 / 2}$ over all $p \in$ $\Pi_{A, N, \nu}$. The mesh $\Lambda_{N, A}$ is designed to approximately minimize $\left\|f-P_{N}^{*} f\right\|_{2,(0, A)}$, over all meshes with the same number of points, when $f \in \mathcal{C}^{\infty}(0, \infty)$ with $\left|f^{(\nu+1)}(s)\right|=$ $E_{\nu+1}(s), s>0$, where $E_{\nu+1}$ is defined as in Theorem 2.3. It achieves this by ensuring that $\left\|f-P_{N}^{*} f\right\|_{2,\left(y_{j-1}, y_{j}\right)}$ is approximately constant for $j=1, \ldots, N+N_{A}$, i.e., by equidistributing the approximation error over the intervals of the mesh, as shown in the proof of the following result in [20].

THEOREM 3.2. Suppose that $f \in \mathcal{C}^{\infty}(0, \infty)$ and $\left|f^{\prime}(s)\right| \leq E_{1}(s),\left|f^{(\nu+1)}(s)\right| \leq$ $E_{\nu+1}(s), s>0$. Then

$$
\left\|f-P_{N}^{*} f\right\|_{2,(0, A)} \leq C_{\nu} \frac{1+\log ^{1 / 2} A}{N^{\nu+1}} .
$$

To form our approximation space on $[\tilde{a}, \tilde{b}]=\left[\tilde{t}_{0}, \tilde{t}_{n}\right]$, we begin by defining

$$
A_{j}:=\min \left\{\alpha \frac{n^{3} N^{\nu+1}}{\cos \theta}, \tilde{t}_{j}-\tilde{t}_{j-1}\right\}
$$


where $\alpha \geq 1$ is an absolute constant which will be determined experimentally and whose value will not effect the asymptotic convergence rates. The reason for our choice of $A_{j}$ will become apparent shortly, in the proof of Theorem 3.3. Clearly $A_{j}$ is bounded independently of $k(b-a)$. As we are primarily concerned with the high-frequency problem, we assume for simplicity that $A_{j} \geq 1, j=1, \ldots, n$, but remark that in the case $A_{j}<1$ for any value of $j$ then we can define $\Lambda_{N, A_{j}}$ to be an appropriate subset of the points $y_{i}$, and this will give similar approximation properties to those achieved using $\Lambda_{N, A_{j}}$ when $A_{j} \geq 1$. For $j=1, \ldots, n$ we define the two meshes $\Omega_{j}^{+}:=\tilde{t}_{j-1}+\Lambda_{N, A_{j}}, \Omega_{j}^{-}:=\tilde{t}_{j}-\Lambda_{N, A_{j}}$. Letting $e_{ \pm}(s):=\mathrm{e}^{ \pm \mathrm{i} s}, s \in \mathbb{R}$, we then define $V_{\Omega_{j}^{+}, \nu}:=\left\{\sigma e_{+}: \sigma \in \Pi_{\Omega_{j}^{+}, \nu}\right\}, V_{\Omega_{j}^{-}, \nu}:=\left\{\sigma e_{-}: \sigma \in \Pi_{\Omega_{j}^{-}, \nu}\right\}$, for $j=1, \ldots, n$, where

$$
\begin{gathered}
\Pi_{\Omega_{j}^{+}, \nu}:=\left\{\sigma \in L_{2}(\mathbb{R}):\left.\sigma\right|_{\left(\tilde{t}_{j-1}+y_{m-1}, \tilde{t}_{j-1}+y_{m}\right)} \text { is a polynomial of degree } \leq \nu,\right. \text { for } \\
\left.m=1, \ldots, N+N_{A_{j}}, \text { and }\left.\sigma\right|_{\mathbb{R} \backslash\left[\tilde{t}_{j-1}, \tilde{t}_{j-1}+A_{j}\right]}=0\right\} \\
\Pi_{\Omega_{j}^{-}, \nu}:=\left\{\sigma \in L_{2}(\mathbb{R}):\left.\sigma\right|_{\left(\tilde{t}_{j}-y_{m}, \tilde{t}_{j}-y_{m-1}\right)} \text { is a polynomial of degree } \leq \nu,\right. \text { for } \\
\left.m=1, \ldots, N+N_{A_{j}}, \text { and }\left.\sigma\right|_{\mathbb{R} \backslash\left[\tilde{t}_{j}-A_{j}, \tilde{t}_{j}\right]}=0\right\}
\end{gathered}
$$

and $y_{0}, \ldots, y_{N_{A_{j}}}$ are the points of the mesh $\Lambda_{N, A_{j}}$. Our approximation space is then $V_{\Omega, \nu}$, the linear span of $\bigcup_{j=1, \ldots, n}\left\{V_{\Omega_{j}^{+}, \nu} \cup V_{\Omega_{j}^{-}, \nu}\right\}$.

Let $(\cdot, \cdot)$ denote the usual inner product on $L_{2}(\mathbb{R}),\left(\chi_{1}, \chi_{2}\right):=\int_{-\infty}^{\infty} \chi_{1}(s) \overline{\chi_{2}}(s) \mathrm{d} s$, $\chi_{1}, \chi_{2} \in L_{2}(\mathbb{R})$. Then our Galerkin method approximation, $\Phi_{N} \in V_{\Omega, \nu}$, is defined by

$$
\left(\Phi_{N}, \rho\right)=\left(\Psi_{\beta}^{\beta_{c}}, \rho\right)+\left(K_{\beta}^{\beta_{c}} \Phi_{N}, \rho\right) \quad \text { for all } \rho \in V_{\Omega, \nu} ;
$$

equivalently,

$$
\Phi_{N}-P_{N} K_{\beta}^{\beta_{c}} \Phi_{N}=P_{N} Q \Psi_{\beta}^{\beta_{c}}
$$

where $P_{N}: L_{2}(\mathbb{R}) \rightarrow V_{\Omega, \nu}$ is the operator of orthogonal projection onto $V_{\Omega, \nu}$. Equation (3.5) can be written explicitly as a system of $M_{N}$ linear algebraic equations, where $M_{N}$, the dimension of $V_{\Omega, \nu}$, i.e., the number of degrees of freedom, is given by

$$
M_{N}=2(\nu+1) \sum_{j=1}^{n}\left(N+N_{A_{j}}\right) .
$$

By (3.3) and (3.4), where $\bar{A}:=\left(A_{1} \ldots A_{n}\right)^{1 / n} \leq\left(A_{1}+\cdots+A_{n}\right) / n$,

$$
M_{N}<(\nu+1) N n\left[3+\frac{2 \log \bar{A}}{q}\right] \leq(\nu+1) N n\left[3+\frac{2}{q} \log \min \left(\frac{\alpha n^{3} N^{\nu+1}}{\cos \theta}, \frac{k(b-a)}{n}\right)\right] .
$$

Using an argument similar to that for the Galerkin method in [20], it can be shown that, provided (3.2) holds, (3.6) is uniquely solvable and

$$
\left\|\left(I-P_{N} K_{\beta}^{\beta_{c}}\right)^{-1}\right\| \leq \frac{\operatorname{Re} \beta_{c}}{\operatorname{Re} \beta_{c}-\left\|\beta-\beta_{c}\right\|_{\infty}},
$$

and thus

$$
\left\|\Phi^{*}-\Phi_{N}\right\|_{2} \leq \frac{\operatorname{Re} \beta_{c}}{\operatorname{Re} \beta_{c}-\left\|\beta-\beta_{c}\right\|_{\infty}}\left\|\Phi^{*}-P_{N} \Phi^{*}\right\|_{2}
$$


There is also a description in [20] of how one can perturb the original problem in such a way that the condition (3.2) on $\beta$ is forced to hold, and the solution of the perturbed problem is arbitrarily close in an arbitrarily large bounded region to the solution of the original problem. In any case, numerical results in [38] suggest that the Galerkin scheme we propose is stable and convergent even when (3.2) does not hold. In this case the bound (3.9) does not apply, however.

It remains to bound $\left\|\Phi^{*}-P_{N} \Phi^{*}\right\|_{2}$, showing that our approximation space is well adapted to approximate $\Phi^{*}$. We introduce $P_{N}^{+}$and $P_{N}^{-}$, the orthogonal projection operators from $L_{2}(\mathbb{R})$ onto $\Pi_{\Omega^{+}, \nu}$ and $\Pi_{\Omega^{-}, \nu}$, respectively, where $\Pi_{\Omega^{ \pm}, \nu}$ denotes the linear span of $\bigcup_{j=1, \ldots, n} \Pi_{\Omega_{j}^{ \pm}, \nu}$. We also define

$$
\begin{aligned}
f_{+}(s) & := \begin{cases}f_{j}^{+}\left(s-\tilde{t}_{j-1}\right), & s \in\left(\tilde{t}_{j-1}, \tilde{t}_{j}\right], j=1, \ldots, n, \\
0, & s \in \mathbb{R} \backslash\left(\tilde{t}_{0}, \tilde{t}_{n}\right],\end{cases} \\
f_{-}(s) & := \begin{cases}f_{j}^{-}\left(\tilde{t}_{j}-s\right), & s \in\left(\tilde{t}_{j-1}, \tilde{t}_{j}\right], j=1, \ldots, n, \\
0, & s \in \mathbb{R} \backslash\left(\tilde{t}_{0}, \tilde{t}_{n}\right] .\end{cases}
\end{aligned}
$$

Then we have the following error estimate.

THEOREM 3.3. If (1.4) holds for some $\epsilon>0$, then

$$
\left\|f_{+}-P_{N}^{+} f_{+}\right\|_{2} \leq C_{\epsilon, \nu} \frac{n^{1 / 2}}{N^{\nu+1}}\left(1+\log ^{1 / 2}\left(\min \left(\alpha \frac{n^{3} N^{\nu+1}}{\cos \theta}, k(b-a)\right)\right)\right),
$$

where $\alpha$ is the constant in (3.4), and the identical bound holds on $\left\|f_{-}-P_{N}^{-} f_{-}\right\|_{2}$.

Proof. We prove the result for $\left\|f_{+}-P_{N}^{+} f_{+}\right\|_{2}$, the bound on $\left\|f_{-}-P_{N}^{-} f_{-}\right\|_{2}$ can be proved in a similar way. Recalling (3.4),

$$
\begin{aligned}
\left\|f_{+}-P_{N}^{+} f_{+}\right\|_{2}^{2} & =\left\|f_{+}-P_{N}^{+} f_{+}\right\|_{2,(\tilde{a}, \tilde{b})}^{2} \\
& =\sum_{j=1}^{n}\left[\left\|f_{+}-P_{N}^{+} f_{+}\right\|_{2,\left(\tilde{t}_{j-1}, \tilde{t}_{j-1}+A_{j}\right)}^{2}+\left\|f_{+}-P_{N}^{+} f_{+}\right\|_{2,\left(\tilde{t}_{j-1}+A_{j}, \tilde{t}_{j}\right)}^{2}\right] .
\end{aligned}
$$

Now, by Theorems 2.3 and 3.2 ,

$$
\left\|f_{+}-P_{N}^{+} f_{+}\right\|_{2,\left(\tilde{t}_{j-1}, \tilde{t}_{j-1}+A_{j}\right)} \leq C_{\epsilon, \nu} \cos \theta \frac{1+\log ^{1 / 2} A_{j}}{N^{\nu+1}} .
$$

If $\alpha n^{3} N^{\nu+1} / \cos \theta \geq \tilde{t}_{j}-\tilde{t}_{j-1}$, then $A_{j}=\tilde{t}_{j}-\tilde{t}_{j-1}$, in which case

$$
\left\|f_{+}-P_{N}^{+} f_{+}\right\|_{2,\left(\tilde{t}_{j-1}+A_{j}, \tilde{t}_{j}\right)}=0 .
$$

If $\alpha n^{3} N^{\nu+1} / \cos \theta<\tilde{t}_{j}-\tilde{t}_{j-1}$, then $A_{j}=\alpha n^{3} N^{\nu+1} / \cos \theta$, and then, recalling the definition of $\Pi_{\Omega^{+}, \nu}$ and Theorem 2.6,

$$
\begin{aligned}
\left\|f_{+}-P_{N}^{+} f_{+}\right\|_{2,\left(\tilde{t}_{j-1}+A_{j}, \tilde{t}_{j}\right)}^{2} & =\left\|f_{+}\right\|_{2,\left(\tilde{t}_{j-1}+A_{j}, \tilde{t}_{j}\right)}^{2} \leq C_{\epsilon} \frac{n^{6}}{\cos ^{2} \theta} \int_{A_{j}}^{\infty} s^{-3} \mathrm{~d} s \\
& =C_{\epsilon} \frac{n^{6}}{2 \cos ^{2} \theta} A_{j}^{-2}=\frac{C_{\epsilon}}{2 \alpha^{2}} N^{-2(\nu+1)},
\end{aligned}
$$

and recalling that $\alpha \geq 1$ the result follows. 
To use the above error estimate, note from (2.16) that $\Phi^{*}=e_{+} f_{+}+e_{-} f_{-}$. But $e_{+} P_{N}^{+} f_{+}+e_{-} P_{N}^{-} f_{-} \in V_{\Omega, \nu}$, and $P_{N} \Phi^{*}$ is the best approximation to $\Phi^{*}$ in $V_{\Omega, \nu}$. So

$$
\begin{aligned}
\left\|\Phi^{*}-P_{N} \Phi^{*}\right\|_{2} & \leq\left\|\Phi^{*}-\left(e_{+} P_{N}^{+} f_{+}+e_{-} P_{N}^{-} f_{-}\right)\right\|_{2} \\
& =\left\|e_{+}\left(f_{+}-P_{N}^{+} f_{+}\right)+e_{-}\left(f_{-}-P_{N}^{-} f_{-}\right)\right\|_{2} \\
& \leq\left\|e_{+}\right\|_{\infty}\left\|f_{+}-P_{N}^{+} f_{+}\right\|_{2}+\left\|e_{-}\right\|_{\infty}\left\|f_{-}-P_{N}^{-} f_{-}\right\|_{2} .
\end{aligned}
$$

Applying Theorem 3.3 we obtain the following result.

THEOREM 3.4. If (1.4) holds for some $\epsilon>0$, then

$$
\left\|\Phi^{*}-P_{N} \Phi^{*}\right\|_{2} \leq C_{\epsilon, \nu} \frac{n^{1 / 2}}{N^{\nu+1}}\left(1+\log ^{1 / 2}\left(\min \left(\alpha \frac{n^{3} N^{\nu+1}}{\cos \theta}, k(b-a)\right)\right)\right),
$$

where $\alpha$ is the constant in (3.4).

Combining this result with the stability bound (3.9) we obtain our final error estimate for the approximation of $\Phi$ by $\Phi_{N}$.

TheOrem 3.5. If (1.4) holds for some $\epsilon>0$, and (3.2) is satisfied, then

$$
\left\|\Phi-\Phi_{N}\right\|_{2,(\tilde{a}, \tilde{b})}=\left\|\Phi^{*}-\Phi_{N}\right\|_{2} \leq \frac{C_{\epsilon, \nu} n^{1 / 2}\left(1+\log ^{1 / 2}\left(\min \left(\alpha n^{3} N^{\nu+1} / \cos \theta, k(b-a)\right)\right)\right)}{\left(\operatorname{Re} \beta_{c}-\left\|\beta-\beta_{c}\right\|_{\infty}\right) N^{\nu+1}},
$$

where $\alpha$ is the constant in (3.4). Further, the number of degrees of freedom $M_{N}$ satisfies

$$
M_{N} \leq C_{\nu} N n\left[1+\log \min \left(\frac{\alpha n^{3} N^{\nu+1}}{\cos \theta}, \frac{k(b-a)}{n}\right)\right] .
$$

We finish by considering the computation of an approximation to $u^{t}$ throughout the upper half plane $U$, once the Galerkin solution $\Phi_{N}$ has been computed. Recalling (2.13) and (2.14) we define $\phi_{N} \in L_{2}(\tilde{a}, \tilde{b})$, an approximation to $\phi$ on $(\tilde{a}, \tilde{b})$, by

$$
\phi_{N}(s):=\Phi_{N}(s)+\psi_{\beta_{j}}(s), \quad s \in\left(\tilde{t}_{j-1}, \tilde{t}_{j}\right], j=1, \ldots, n,
$$

where $\psi_{\beta_{j}}$ is given explicitly by (2.7). Then, recalling that $u^{t}\left(\left(y_{1}, 0\right)\right)=\phi\left(k y_{1}\right)$, we define an approximation to $u^{t}$ by replacing $u^{t}(y)$ by its approximation $\phi_{N}\left(k y_{1}\right)$ in (2.5), to give the approximation $u_{N}^{t}$ defined by

$$
u_{N}^{t}(x):=u_{\beta_{c}}^{t}(x)+\mathrm{i} k \int_{a}^{b} G_{\beta_{c}}\left(x,\left(y_{1}, 0\right)\right)\left(\beta\left(y_{1}\right)-\beta_{c}\right) \phi_{N}\left(k y_{1}\right) \mathrm{d} y_{1} .
$$

From (2.2) and (2.3), and using properties of standard single-layer potentials [23], it follows that $u_{N}^{t} \in C^{2}(U) \cap C(\bar{U})$ and satisfies the Helmholtz equation (1.1) in $U$. Further, from Theorem 3.5 we deduce the following error estimate.

THEOREM 3.6. If (1.4) holds for some $\epsilon>0$, and (3.2) is satisfied, then

$$
\left|u^{t}(x)-u_{N}^{t}(x)\right| \leq \frac{C_{\epsilon, \nu} n^{1 / 2}\left(1+\log ^{1 / 2}\left(\min \left(\alpha n^{3} N^{\nu+1} / \cos \theta, k(b-a)\right)\right)\right)}{\left(\operatorname{Re} \beta_{c}-\left\|\beta-\beta_{c}\right\|_{\infty}\right) N^{\nu+1}}
$$

for $x \in \bar{U}$, where $\alpha$ is the constant in (3.4). 
Proof. Subtracting (3.10) from (2.5) and using the Cauchy-Schwarz inequality and the definitions of $\Phi^{*}$ and $\phi_{N}$, we see that

$$
\begin{aligned}
\left|u^{t}(x)-u_{N}^{t}(x)\right| & =\left|\int_{\tilde{a}}^{\tilde{b}} G_{\beta_{c}}(x,(t / k, 0))\left(\beta(t / k)-\beta_{c}\right)\left(\Phi(t)-\Phi_{N}(t)\right) \mathrm{d} t\right| \\
& \leq\left\|\beta-\beta_{c}\right\|_{\infty}\left\{\int_{-\infty}^{\infty}\left|G_{\beta_{c}}(x,(t / k, 0))\right|^{2} \mathrm{~d} t\right\}^{1 / 2}\left\|\Phi-\Phi_{N}\right\|_{2,(\tilde{a}, \tilde{b})} .
\end{aligned}
$$

Now, defining $H=k x_{2}$ and using (2.2) we see that for $H \geq 1 / 2$ it holds that

$$
\begin{aligned}
\int_{-\infty}^{\infty}\left|G_{\beta_{c}}(x,(t / k, 0))\right|^{2} \mathrm{~d} t & \leq C_{\epsilon}(1+H)^{2} \int_{-\infty}^{\infty} \frac{\mathrm{d} t}{\left(t^{2}+H^{2}\right)^{3 / 2}} \\
& =2 C_{\epsilon} \frac{(1+H)^{2}}{H^{2}} \int_{0}^{\infty} \frac{\mathrm{d} s}{\left(1+s^{2}\right)^{3 / 2}} \leq C_{\epsilon} \int_{0}^{\infty} \frac{\mathrm{d} s}{\left(1+s^{2}\right)^{3 / 2}} .
\end{aligned}
$$

Using (2.2) and (2.3) we see that, for $0 \leq H<1 / 2$,

$$
\begin{aligned}
\int_{-\infty}^{\infty}\left|G_{\beta_{c}}(x,(t / k, 0))\right|^{2} \mathrm{~d} t & \leq C_{\epsilon}\left(\int_{\sqrt{1-H^{2}}}^{\infty} \frac{(1+H)^{2} \mathrm{~d} t}{\left(t^{2}+H^{2}\right)^{3 / 2}}+\int_{0}^{\sqrt{1-H^{2}}}\left(1-\frac{1}{2} \log \left(t^{2}+H^{2}\right)\right) \mathrm{d} t\right) \\
& \leq C_{\epsilon}\left(\frac{9}{4} \int_{\sqrt{3} / 2}^{\infty} \frac{\mathrm{d} t}{t^{3}}+\int_{0}^{1}(1-\log t) \mathrm{d} t\right) .
\end{aligned}
$$

Thus $\left|u^{t}(x)-u_{N}^{t}(x)\right| \leq C_{\epsilon}\left\|\Phi-\Phi_{N}\right\|_{2,(\tilde{a}, \tilde{b})}$, and the result follows from Theorem 3.5.

4. Implementation and numerical results. We restrict our attention in this section to the case $\nu=0$. The implementation of the scheme is similar for higher values of $\nu$. Recalling (3.5), the equation we wish to solve is

$$
\left(\Phi_{N}, \rho\right)-\left(K_{\beta}^{\beta_{c}} \Phi_{N}, \rho\right)=\left(\Psi_{\beta}^{\beta_{c}}, \rho\right) \quad \text { for all } \rho \in V_{\Omega, 0} .
$$

Writing $\Phi_{N}$ as a linear combination of basis functions of $V_{\Omega, 0}$, we have $\Phi_{N}(s)=$ $\sum_{j=1}^{M_{N}} v_{j} \rho_{j}(s)$, where $M_{N}$ is given by (3.7) and $\rho_{j}$ is the $j$ th basis function, defined by

$$
\begin{aligned}
& \rho_{j}(s):=\frac{\mathrm{e}^{\mathrm{i} s} \chi_{\left[s_{\tilde{j}}^{+}, s_{\tilde{j}-1}^{+}\right)}^{+}(s)}{\left(s_{\tilde{j}}^{+}-s_{\tilde{j}-1}^{+}\right)^{1 / 2}}, \quad j=\tilde{j}+2 \sum_{m=1}^{p-1}\left(N+N_{A_{m}}\right), \tilde{j}=1, \ldots, N+N_{A_{p}}, \\
& \rho_{j}(s):=\frac{\mathrm{e}^{-\mathrm{i} s} \chi_{\left[s_{\tilde{j}}^{-}, s_{\tilde{j}-1}^{-}\right)}(s)}{\left(s_{\tilde{j}}^{-}-s_{\tilde{j}-1}^{-}\right)^{1 / 2}}, \quad j=\tilde{j}+N+N_{A_{p}}+2 \sum_{m=1}^{p-1}\left(N+N_{A_{m}}\right), \tilde{j}=1, \ldots, N+N_{A_{p}},
\end{aligned}
$$

for $p=1, \ldots, n$, where $s_{l}^{+} \in \Omega_{p}^{+}, s_{l}^{-} \in \Omega_{p}^{-}$for $l=0, \ldots, N+N_{A_{p}}$, and $\chi_{\left[s_{1}, s_{2}\right)}$ denotes the characteristic function of the interval $\left[s_{1}, s_{2}\right.$ ). Equation (4.1) then becomes the linear system

$$
\sum_{j=1}^{M_{N}} v_{j}\left(\left(\rho_{j}, \rho_{m}\right)-\left(K_{\beta}^{\beta_{c}} \rho_{j}, \rho_{m}\right)\right)=\left(\Psi_{\beta}^{\beta_{c}}, \rho_{m}\right), \quad m=1, \ldots, M_{N} .
$$

If $k$ is large compared to $N$, then, from the definition of $A_{j}$ in (3.4), it is clear that the two meshes $\Omega_{j}^{+}$and $\Omega_{j}^{-}$will not overlap. In this case the basis functions $\rho_{j}$, 
$j=1, \ldots, M_{N}$, form an orthonormal basis for $V_{\Omega, \nu}$ (this is not true for the Galerkin method described in [20]), and hence the condition number of our linear system (4.2) will be bounded by (see, e.g., [2, section 3.6.3])

$$
\begin{aligned}
\left\|\left(I-P_{N} K_{\beta}^{\beta_{c}}\right)\right\|_{2}\left\|\left(I-P_{N} K_{\beta}^{\beta_{c}}\right)^{-1}\right\|_{2} & \leq\left(1+\left\|K_{\beta}^{\beta_{c}}\right\|_{2}\right)\left(\frac{\operatorname{Re} \beta_{c}}{\operatorname{Re} \beta_{c}-\left\|\beta-\beta_{c}\right\|_{\infty}}\right) \\
& \leq\left(1+\frac{\left\|\beta-\beta_{c}\right\|_{\infty}}{\operatorname{Re} \beta_{c}}\right)\left(\frac{\operatorname{Re} \beta_{c}}{\operatorname{Re} \beta_{c}-\left\|\beta-\beta_{c}\right\|_{\infty}}\right) \\
& =\frac{\operatorname{Re} \beta_{c}+\left\|\beta-\beta_{c}\right\|_{\infty}}{\operatorname{Re} \beta_{c}-\left\|\beta-\beta_{c}\right\|_{\infty}},
\end{aligned}
$$

where we have used (3.8) (under the assumption that (3.2) holds) and the facts that $\left\|K_{\beta}^{\beta_{c}}\right\|_{2} \leq\left\|\beta-\beta_{c}\right\|_{\infty} / \operatorname{Re} \beta_{c}$ (see, e.g., $\left.[20,(3.2)]\right)$ and $\left\|P_{N}\right\|_{2}=1$. The fact that we can establish such a bound on the condition number of our linear system is in direct contrast to some other schemes in the literature where the approximation space consists of plane wave basis functions, e.g., [41, 44, 45], where serious difficulties due to ill-conditioning have been reported.

To evaluate the coefficients $\left(K_{\beta}^{\beta_{c}} \rho_{j}, \rho_{m}\right)$ and $\left(\Psi_{\beta}^{\beta_{c}}, \rho_{m}\right)$ of (4.2) we must compute some integrals numerically. The exact formulas are given in [38], but note that after some integrations are carried out analytically, the most difficult of these take the forms

$$
\int_{0}^{\infty} \frac{(\mathrm{i}-r) F(r)}{r(r-2 \mathrm{i})} \mathrm{d} r, \int_{0}^{\infty} \frac{\left(1-\mathrm{e}^{r s}\right) F(r)}{r^{2}} \mathrm{~d} r, \int_{0}^{\infty} \frac{\left(1-\mathrm{e}^{r s}\right) F(r)}{r(r-2 \mathrm{i})} \mathrm{d} r
$$

where $s<0$ and $F(r)$ is given by (2.20). These integrals are similar in difficulty to integral representations for the Green's function $G_{\beta^{*}}$, for which very efficient numerical schemes are proposed in [18]. The integrands are not oscillatory and the coefficients do not become more difficult to evaluate as $k \rightarrow \infty$.

As a numerical example, we take $\theta=\pi / 4, n=1$, and

$$
\beta(s)= \begin{cases}0.505-0.3 \mathrm{i}, & s \in[-m \lambda, m \lambda], \\ 1, & s \notin[-m \lambda, m \lambda],\end{cases}
$$

for $m=5,10,20,40,80,160,320,640,1280,2560$, and 5120, where $k=1$ and $\lambda=2 \pi$ is the wavelength. This experiment is equivalent to fixing the interval $[a, b]=$ $\left[t_{0}, t_{1}\right]$ and decreasing the wavelength. The assumption (3.2) is satisfied, so that Theorem 3.5 holds. For each value of $m$, we compute $\Phi_{N}$ with $\nu=0, \alpha=25 \sqrt{2}$ (so that $\alpha n^{3} / \cos \theta=\sqrt{2} \alpha=50$, this value chosen experimentally) and $N=2,4$, $8,16,32,64$. For the purpose of computing errors, we take the "exact" solution $\left(\Phi^{*}\right)$ to be the solution computed with $\sqrt{2} \alpha=1000$ and $N=128$. Whereas for the scheme of [20] the number of degrees of freedom needed to maintain accuracy increases logarithmically with respect to $k(b-a)$ as $k(b-a) \rightarrow \infty$, here the number needed to maintain accuracy remains bounded as $k(b-a) \rightarrow \infty$, as we shall see below.

In Figure 4.1 we plot $\left|\Phi^{*}\right|$ and $\left|\Phi_{2}\right|$ for $m=10$. Noting the logarithmic scales on the plots, it is clear that $\left|\Phi^{*}\right|$ is highly peaked near the discontinuities in impedance. Recalling that $\Phi$ is a correction term, namely, the difference between the true solution and the solution that there would be if the impedance were constant everywhere, the reason for this is clear. On the plot of $\left|\Phi_{2}\right|$ we also show the two grids $\Omega_{1}^{+}$and $\Omega_{1}^{-}$. For 

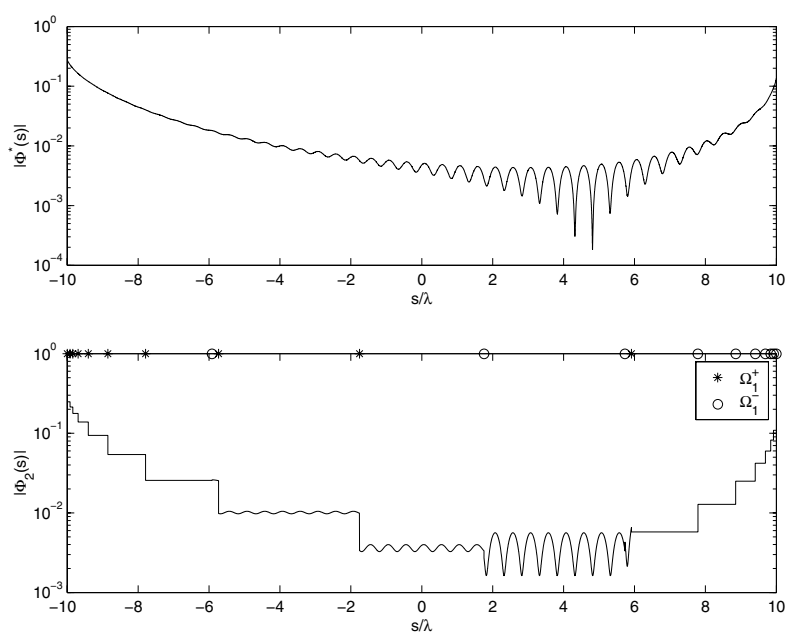

FIG. 4.1. Plot of $\left|\Phi^{*}\right|$ and $\left|\Phi_{2}\right|, m=10$, so that $b-a=20 \lambda$.
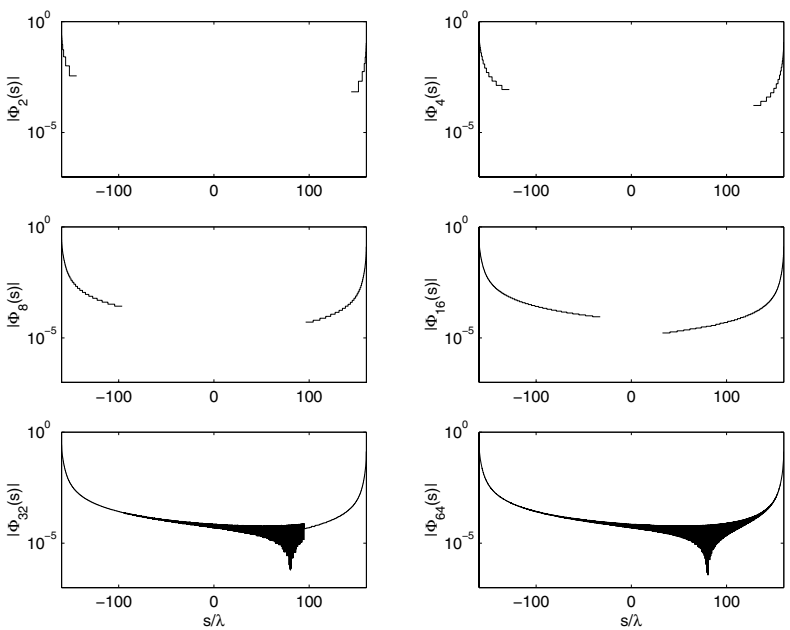

Fig. 4.2. Plot of $\left|\Phi_{N}\right|, N=2,4,8,16,32,64$ for $m=160$, so that $b-a=320 \lambda$.

$s / \lambda$ less than about -6 and for $s / \lambda$ greater than about 6 the grids do not overlap, and on these regions $\Phi_{2}(s)=e^{\mathrm{i} s} \times$ (piecewise constants) and $\Phi_{2}(s)=e^{-\mathrm{i} s} \times$ (piecewise constants), respectively. Thus $\left|\Phi_{2}(s)\right|$ is piecewise constant where the grids do not overlap, and this can be clearly seen in Figure 4.1. Where the grids overlap, (roughly between $s / \lambda=-6$ and $s / \lambda=6)$ the oscillatory nature of $\Phi_{2}(s)$ is more apparent.

In Figure 4.2 we plot $\left|\Phi_{N}\right|$ for $m=160$ and for $N=2,4,8,16,32,64$. Again noting the logarithmic scales on each plot, $\left|\Phi_{N}\right|$ is highly peaked near the impedance discontinuities, much more so than for $m=10$. As $N$ increases so we discretize a larger part of the domain $[-m \lambda, m \lambda]$, as well as having a finer mesh near the discontinuities in impedance at $-m \lambda, m \lambda$. For $N=2,4,8,16$ the piecewise constant approximation can be clearly seen, as the grids $\Omega_{1}^{+}$and $\Omega_{1}^{-}$do not overlap. For $N=32$ the grids overlap between about $s \lambda=-100$ and $s \lambda=100$. For $N=64$, each grid covers the whole domain $[-m \lambda, m \lambda]$. 

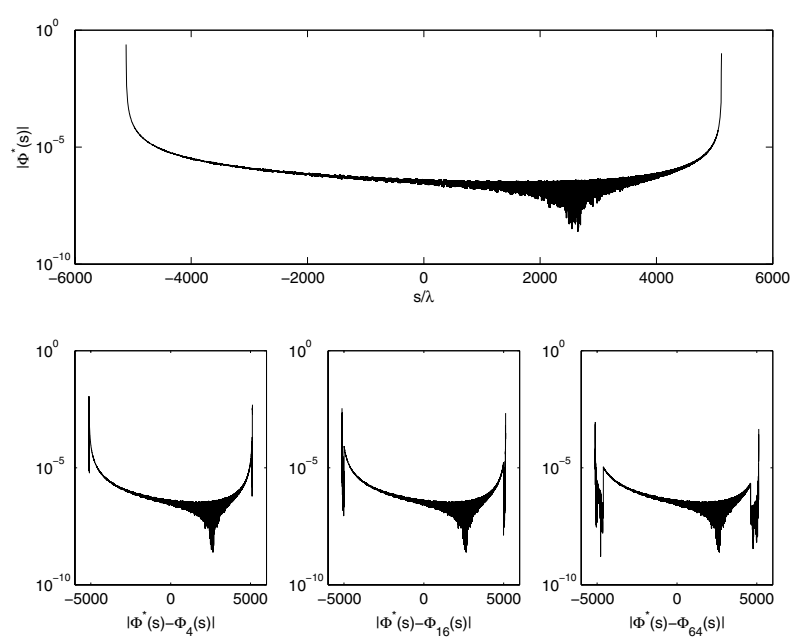

FIG. 4.3. Plot of $\left|\Phi^{*}\right|$ and $\left|\Phi^{*}-\Phi_{N}\right|, N=4,16,64$ for $m=5120$, so that $b-a=10240 \lambda$.

TABLE 4.1

$\left\|\Phi^{*}-\Phi_{N}\right\|_{2} /\left\|\Phi^{*}\right\|_{2}$ for $m=10,160$, and 5120 , and increasing $N$.

\begin{tabular}{|r|r|r|r|r|r|}
\hline$(b-a) / \lambda$ & $N$ & $M_{N}$ & $\left\|\Phi^{*}-\Phi_{N}\right\|_{2} /\left\|\Phi^{*}\right\|_{2}$ & EOC & COND \\
\hline 20 & 2 & 18 & $1.635 \times 10^{-1}$ & 1.1 & 1.8 \\
& 4 & 42 & $7.393 \times 10^{-2}$ & 1.1 & 2.6 \\
& 8 & 90 & $3.525 \times 10^{-2}$ & 1.0 & 8.1 \\
& 16 & 182 & $1.773 \times 10^{-2}$ & 1.0 & 94.0 \\
& 32 & 370 & $8.875 \times 10^{-3}$ & 1.0 & 625.5 \\
& 64 & 742 & $4.557 \times 10^{-3}$ & & 2551.6 \\
\hline 320 & 2 & 18 & $1.647 \times 10^{-1}$ & 1.2 & 1.8 \\
& 4 & 46 & $7.399 \times 10^{-2}$ & 1.0 & 2.0 \\
& 8 & 106 & $3.622 \times 10^{-2}$ & 1.0 & 2.0 \\
& 16 & 240 & $1.790 \times 10^{-2}$ & 1.0 & 2.1 \\
& 32 & 530 & $8.662 \times 10^{-3}$ & 0.9 & 2.1 \\
& 64 & 1094 & $4.537 \times 10^{-3}$ & & 92.7 \\
\hline 10240 & 2 & 18 & $1.639 \times 10^{-1}$ & 1.2 & 1.8 \\
& 4 & 46 & $6.918 \times 10^{-2}$ & 0.8 & 2.0 \\
& 8 & 106 & $3.881 \times 10^{-2}$ & 1.2 & 2.0 \\
& 16 & 240 & $1.751 \times 10^{-2}$ & 1.1 & 2.1 \\
& 32 & 530 & $8.076 \times 10^{-3}$ & 0.8 & 2.1 \\
& 64 & 1154 & $4.579 \times 10^{-3}$ & & 2.1 \\
\hline
\end{tabular}

In Figure 4.3 we plot $\left|\Phi^{*}\right|$ and $\left|\Phi^{*}-\Phi_{N}\right|$ for $m=5120$ and for $N=4,16$ and 64 . In this case the interval $[-m \lambda, m \lambda]$ is over 10,000 wavelengths long, and so even for $N=64$ the grids $\Omega_{1}^{+}$and $\Omega_{1}^{-}$do not overlap. As $m$ increases, so $\left|\Phi^{*}\right|$ becomes even more peaked, and the benefit of clustering the grid points around the impedance discontinuities becomes even more apparent.

For $m=10,160$, and 5120 the relative $L_{2}$ errors $\left\|\Phi^{*}-\Phi_{N}\right\|_{2} /\left\|\Phi^{*}\right\|_{2}$ are shown in Table 4.1. (All $L_{2}$ norms are computed by approximating by discrete $L_{2}$ norms, sampling at 100,000 evenly spaced points in the relevant interval for the function whose norm is to be evaluated.) The estimated order of convergence is given by

$$
\mathrm{EOC}:=\log _{2}\left(\frac{\left\|\Phi^{*}-\Phi_{N}\right\|_{2}}{\left\|\Phi^{*}-\Phi_{2 N}\right\|_{2}}\right)
$$


TABLE 4.2

$\left\|\Phi^{*}-\Phi_{16}\right\|_{2} /\left\|\Phi^{*}\right\|_{2}$ for increasing interval length.

\begin{tabular}{|r|r|r|r|r|}
\hline$(b-a) / \lambda$ & $M_{N}$ & $\left\|\Phi^{*}-\Phi_{16}\right\|_{2} /\left\|\Phi^{*}\right\|_{2}$ & $\left\|\Phi^{*}-\Phi_{16}\right\|_{2}$ & COND \\
\hline 10 & 162 & $1.746 \times 10^{-2}$ & $7.936 \times 10^{-3}$ & 181.5 \\
20 & 182 & $1.773 \times 10^{-2}$ & $8.059 \times 10^{-3}$ & 94.0 \\
40 & 204 & $1.775 \times 10^{-2}$ & $8.068 \times 10^{-3}$ & 24.7 \\
80 & 226 & $1.766 \times 10^{-2}$ & $8.027 \times 10^{-3}$ & 8.2 \\
160 & 240 & $1.761 \times 10^{-2}$ & $8.000 \times 10^{-3}$ & 2.1 \\
320 & 240 & $1.790 \times 10^{-2}$ & $8.122 \times 10^{-3}$ & 2.1 \\
640 & 240 & $1.749 \times 10^{-2}$ & $7.916 \times 10^{-3}$ & 2.1 \\
1280 & 240 & $1.650 \times 10^{-2}$ & $7.435 \times 10^{-3}$ & 2.1 \\
2560 & 240 & $1.616 \times 10^{-2}$ & $7.216 \times 10^{-3}$ & 2.1 \\
5120 & 240 & $1.556 \times 10^{-2}$ & $6.831 \times 10^{-3}$ & 2.1 \\
10240 & 240 & $1.751 \times 10^{-2}$ & $7.433 \times 10^{-3}$ & 2.1 \\
\hline
\end{tabular}

For this example, Theorem 3.5 predicts that

$$
\left\|\Phi^{*}-\Phi_{N}\right\|_{2} \leq \frac{C}{N}\left(1+\log ^{1 / 2}(\min (\sqrt{2} \alpha N, 2 m \lambda))\right),
$$

so that we expect $\mathrm{EOC} \approx 1$, and this is what we see. For each value of $m$, the number of degrees of freedom $M_{N}$ increases approximately in proportion to $N \log N$ as $N$ increases until the two grids $\Omega_{1}^{+}$and $\Omega_{1}^{-}$each cover the whole domain $[-m \lambda, m \lambda]$ (i.e., until $\sqrt{2} \alpha N \geq 2 m \lambda$ ), after which $M_{N}$ increases only proportionally to $N$ as $N$ increases further. For $m=10$, the whole domain is covered by the grids for $N=4$; for $m=160$ this occurs for $N=64$ but for $m=5120$ the two grids do not overlap even for $N=64$. The condition numbers for the matrix of the linear system (4.2) (denoted by COND) satisfy the bound (4.3), which predicts that COND $\leq 3.75$ if the grids do not overlap, i.e., if $N \leq 16$ for $m=160$, for all values of $N$ when $m=5120$. For $N \leq 32$ the number of degrees of freedom is the same for $m=160$ and $m=5120$, and yet the relative $L_{2}$ error is almost the same for the two cases $b-a=320 \lambda$ and $b-a=10240 \lambda$.

In Table 4.2 we fix $N=16$ and show $\left\|\Phi^{*}-\Phi_{16}\right\|_{2} /\left\|\Phi^{*}\right\|_{2}$ and also $\left\|\Phi^{*}-\Phi_{16}\right\|_{2}$ for increasing values of $m=(b-a) / 2 \lambda$. As $m$ increases, the number of degrees of freedom increases logarithmically for those values of $m$ for which $\sqrt{2} \alpha N \geq 2 m \lambda$, i.e., for $m \leq 40$, but as $m$ increases further for $m \geq 80$ the number of degrees of freedom remains constant, and yet both the relative and the actual $L_{2}$ error also remain roughly constant as $m$ grows. For $m=5120$ the interval is of length greater than 10,000 wavelengths, and yet we achieve almost $1 \%$ relative error with only 240 degrees of freedom. As in Table 4.1, the condition number of the linear system (4.2) is bounded by (4.3), so that COND $\leq 3.75$, when $m$ is sufficiently large that the grids $\Omega_{1}^{+}$and $\Omega_{1}^{-}$do not overlap, i.e., for $m \geq 160$.

In the last figure and table we show numerical computations of the total field above the boundary, i.e., $u_{N}^{t}(x)$ given by (3.10). We note that computing $u_{N}^{t}(x)$ requires, for each point $x$, the computation of the highly oscillatory integral (3.10), which is evaluated here using accurate but slow "black box" techniques. In the future it is hoped that more efficient quadrature schemes can be developed, taking advantage of the fact that the oscillatory parts of both $G_{\beta_{c}}$ and $\phi_{N}$ are known explicitly. We note that Iserles $[34,35]$ has recently proposed and analyzed Filon-type quadrature methods appropriate for the efficient evaluation of highly oscillatory integrals, which we expect may be appropriate. 

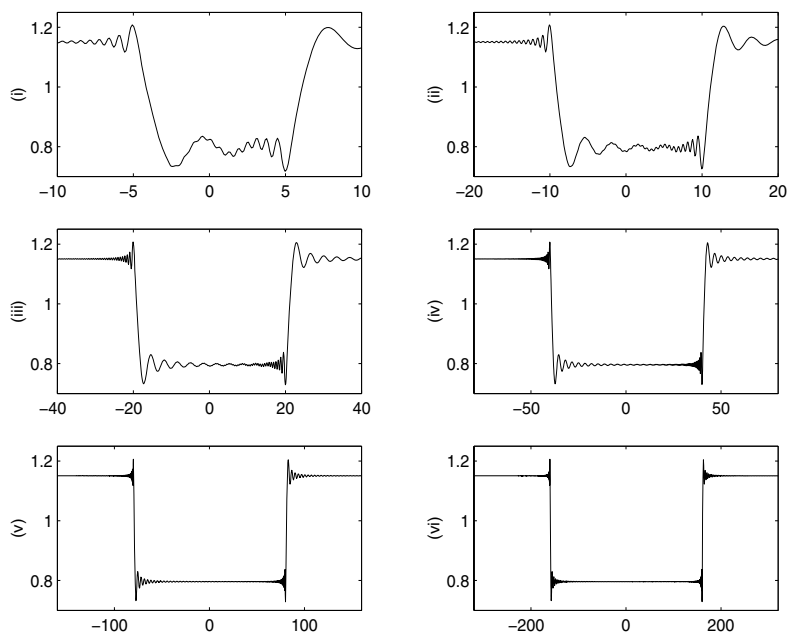

FIG. 4.4. $\left|u^{t}(x)\right|$ (on the $y$-axis) against $x_{1} / \lambda$ (on the $x$-axis) for $x=\left(x_{1}, \lambda\right), x_{1} \in[-2 m \lambda, 2 m \lambda]$, plotted for $m=5$ (plot (i)), $m=10$ (plot (ii)), $m=20$ (plot (iii)), $m=40$ (plot (iv)), $m=80$ (plot (v)), and $m=160$ (plot (vi)).

TABLE 4.3

$\left|u^{t}(x)-u_{N}^{t}(x)\right|$ for $m=10$ and $m=160$, and increasing $N$.

\begin{tabular}{|r|r|r|r|r|r|}
\hline & & \multicolumn{2}{|c|}{$x=(m \lambda / 2, \lambda)$} & \multicolumn{2}{c|}{$x=(m \lambda, \lambda)$} \\
\hline$m$ & $N$ & $\left|u^{t}(x)-u_{N}^{t}(x)\right|$ & EOC & $\left|u^{t}(x)-u_{N}^{t}(x)\right|$ & EOC \\
\hline 10 & 2 & $3.894 \times 10^{-4}$ & 1.5 & $1.108 \times 10^{-4}$ & 1.3 \\
& 4 & $1.421 \times 10^{-4}$ & 2.5 & $4.514 \times 10^{-5}$ & 3.5 \\
& 8 & $2.432 \times 10^{-5}$ & 1.0 & $4.068 \times 10^{-6}$ & 0.2 \\
& 16 & $1.183 \times 10^{-5}$ & 2.7 & $3.448 \times 10^{-6}$ & 0.8 \\
& 32 & $1.841 \times 10^{-6}$ & 1.0 & $2.014 \times 10^{-6}$ & 1.1 \\
& 64 & $9.350 \times 10^{-7}$ & & $9.108 \times 10^{-7}$ & \\
\hline 160 & 2 & $1.059 \times 10^{-4}$ & 2.0 & $5.278 \times 10^{-4}$ & 2.6 \\
& 4 & $2.572 \times 10^{-5}$ & 0.4 & $8.790 \times 10^{-5}$ & 2.8 \\
& 8 & $1.978 \times 10^{-5}$ & 0.0 & $1.283 \times 10^{-5}$ & 0.3 \\
& 16 & $1.981 \times 10^{-5}$ & 2.1 & $1.060 \times 10^{-5}$ & 0.8 \\
& 32 & $4.474 \times 10^{-6}$ & 0.9 & $6.029 \times 10^{-6}$ & 3.4 \\
& 64 & $2.431 \times 10^{-6}$ & & $5.634 \times 10^{-7}$ & \\
\hline
\end{tabular}

In Figure 4.4 we plot $\left|u_{128}^{t}(x)\right|$ for $x=\left(x_{1}, \lambda\right), x_{1} \in[-2 m \lambda, 2 m \lambda]$, i.e., the absolute value of the total acoustic field one wavelength above the plane, as computed with $\sqrt{2} \alpha=1000$ and $N=128$, for $m=5$ (plot (i)), $m=10$ (plot (ii)), $m=20$ (plot (iii)), $m=40$ (plot (iv)), $m=80$ (plot (v)), and $m=160$ (plot (vi)). In each plot the $x$-axis represents $x_{1} / \lambda$ and the $y$-axis represents $\left|u^{t}(x)\right|$. One can clearly see that the wave diffracted from the impedance discontinuities at $x=(-m \lambda, 0)$ and $x=(m \lambda, 0)$ is a significant component of the total field only within a small number of wavelengths of the impedance discontinuities. Figure 1.1 shows a surface plot of the incident, scattered and total wave fields up to 10 wavelengths above the plane for this same example with $m=5$.

We also computed $u_{N}^{t}(x)$ for $x=(m \lambda / 2, \lambda)$ and $x=(m \lambda, \lambda)$ for $m=10$ and $m=160$ and for $\sqrt{2} \alpha=50, N=2,4,8,16,32$, and 64 . Taking the values for $\alpha=500 \sqrt{2}, N=128$ to be the "exact" values, the errors are shown in Table 4.3. The 
estimated order of convergence is calculated as

$$
\text { EOC }:=\log _{2}\left(\frac{\left|u^{t}(x)-u_{N}^{t}\right|}{\left|u^{t}(x)-u_{2 N}^{t}\right|}\right)
$$

and from Theorem 3.6 we would expect $\mathrm{EOC} \approx 1$. The convergence rate is rather irregular, but broadly speaking it is at least as good as expected, and the actual and relative errors are both very small. At every point $x$ it holds that $0.7<\left|u^{t}(x)\right|<0.9$.

Further numerical results for $\theta \approx \pi / 2$, i.e., grazing incidence, can be found in [39].

5. Conclusions and discussion. In this paper we have presented a Galerkin boundary element method for an acoustic scattering problem, and we have demonstrated, via both an a priori error analysis and numerical examples, that the number of degrees of freedom required for an accurate solution is bounded independently of the wavenumber. Our numerical method and analysis are for a specific scattering problem, namely the $2 \mathrm{D}$ problem of scattering by an unbounded flat surface with piecewise constant surface impedance, this problem being important in the theory of outdoor noise propagation and in an electromagnetic context.

As we discussed in our review of the literature, our method is an instance of the general idea of expressing the solution of the scattering problem as a finite sum of known oscillatory terms (given by the leading order behavior of the solution as $k \rightarrow \infty)$ multiplied by unknown more slowly oscillating terms, these smoother components much more suitable for approximation by standard finite element functions than the original solution. Our results add to the evidence of the theory and numerical experiments of other authors $[1,29,24,10,12]$ that this general methodology has promise for a range of scattering problems.

Specifically, we anticipate that many of the details of our numerical scheme and analysis will be applicable to other interesting scattering problems. This is clearest in the case of $2 \mathrm{D}$ acoustic scattering by a convex polygon, in the case that a homogeneous Dirichlet condition or an impedance boundary condition with constant impedance holds on each side. For this problem we expect that the behavior of the total field on each side of the polygon (after subtraction of the leading order high frequency asymptotics given by physical optics) will be very similar to the behavior quantified in Theorems 2.3 and 2.6. Thus the same mesh may be applicable and much of the same analysis. For more discussion of scattering by a 2 D polygon see [20, section 6$]$, [19].

Moreover, we expect that our mesh design will be relevant more generally, at least for representing certain components of the total field. In the case of threedimensional scattering by convex polyhedra it seems to us likely that the mesh we propose will be useful in representing the variation of edge scattered waves in the direction perpendicular to the edge. In the case of $2 \mathrm{D}$ convex curvilinear polygons something close to the mesh we use on each interval $\left[t_{j-1}, t_{j}\right]$ may be appropriate on each side of the polygon, especially at higher frequencies when our mesh becomes more localized near the ends of the intervals just as the waves diffracted by the corners become more localized near the corners.

Acknowledgment. Some of this work was carried out while the authors participated in the 2003 Programme Computational Challenges in Partial Differential Equations at the Isaac Newton Institute, Cambridge, UK. 


\section{REFERENCES}

[1] T. Abboud, J. C. NÉdÉlec, And B. Zhou, Méthodes des équations intégrales pour les hautes fréquences, C.R. Acad. Sci. I Math., 318 (1994), pp. 165-170.

[2] K. E. AtKinson, The Numerical Solution of Integral Equations of the Second Kind, Cambridge University Press, Cambridge, UK, 1997.

[3] K. Attenborough, Acoustical impedance models for outdoor ground surfaces, J. Sound Vib., 99 (1985), pp. 521-544.

[4] I. BabušKa AND J. Melenk, The partition of unity method, Internat. J. Numer. Meth. Engrg., 40 (1997), pp. $727-758$

[5] I. BABUŠKA AND S. SAUTER, Is the pollution effect of the FEM avoidable for the Helmholtz equation considering high wave numbers, SIAM J. Numer. Anal., 34 (1997), pp. 23922423.

[6] F. X. Becot, P. J. Thorsson, And W. Kropp, An efficient application of equivalent sources to noise propagation over inhomogeneous ground, Acta Acoust. United Ac., 88 (2002), pp. 853-860.

[7] P. Boulanger, K. Attenborough, And Q. Qin, Effective impedance of surfaces with porous roughness: Models and data, J. Acoust. Soc. Am., 117 (2005), pp. 1146-1156.

[8] P. Boulanger, K. Attenborough, S. Taherzadeh, T. WatersFuller, and K. M. Li, Ground effect over hard rough surfaces, J. Acoust. Soc. Am., 104 (1998), pp. 1474-1482.

[9] O. BRuno And L. Kunyansky, A fast, high-order algorithm for the solution of surface scattering problems: Basic implementation, tests and applications, J. Comput. Phys., 169 (2001), pp. $80-110$.

[10] O. P. Bruno, C. A. Geuzaine, J. A. Monro, Jr., And F. Reitich, Prescribed error tolerances within fixed computational times for scattering problems of arbitrarily high frequency: The convex case, Philos. Trans. R. Soc. London A, 362 (2004), pp. 629-645.

[11] O. P. Bruno, C. A. Geuzaine, and F. Reitich, A new high-order high-frequency integral equation method for the solution of scattering problems i: Single-scattering configurations, in Proceedings of the 20th Annual Review of Progress in Applied Computational Electromagnetics, Syracuse, NY, 2004.

[12] O. P. Bruno, C. A. Geuzaine, And F. Reitich, A new high-order high-frequency integral equation method for the solution of scattering problems ii: Multiple-scattering configurations, Proceedings of the 20th Annual Review of Progress in Applied Computational Electromagnetics, Syracuse, NY, 2004.

[13] O. Cessenat And B. Després, Application of an ultra weak variational formulation of elliptic PDEs to the two-dimensional Helmholtz problem, SIAM J. Numer. Anal., 35 (1998), pp. $255-299$.

[14] O. Cessenat And B. Després, Using plane waves as base functions for solving time harmonic equations with the ultra weak variational formulation, J. Comp. Acoust., 11 (2003), pp. 227-238.

[15] S. N. Chandler-Wilde, Ground Effects in Environmental Sound Propagation, Ph.D. thesis, University of Bradford, UK, 1988.

[16] S. N. Chandler-Wilde, The impedance boundary value problem for the Helmholtz equation in a half-plane, Math. Methods Appl. Sci., 20 (1997), pp. 813-840.

[17] S. N. Chandler-Wilde And D. C. Hothersall, Sound propagation above an inhomogeneous impedance plane, J. Sound Vib., 98 (1985), pp. 475-491.

[18] S. N. Chandler-Wilde And D. C. Hothersall, Efficient calculation of the Green's function for acoustic propagation above a homogeneous impedance plane, J. Sound Vib., 180 (1995), pp. $705-724$.

[19] S. N. Chandler-Wilde and S. Langdon, A Galerkin boundary element method for high frequency scattering by convex polygons (in preparation).

[20] S. N. Chandler-Wilde, S. Langdon, And L. RitTer, A high-wavenumber boundary-element method for an acoustic scattering problem, Philos. Trans. R. Soc. London A, 362 (2004), pp. 647-671.

[21] S. N. Chandler-Wilde, M. Rahman, and C. R. Ross, A fast two-grid and finite section method for a class of integral equations on the real line with application to an acoustic scattering problem in the half-plane, Numer. Math., 93 (2002), pp. 1-51.

[22] S. H. Christiansen And J. C. NÉDÉLec, Preconditioners for the numerical solution of boundary integral equations from electromagnetism, C.R. Acad. Sci. I Math., 331 (2000), pp. 733738.

[23] D. Colton and R. Kress, Integral Equation Methods in Scattering Theory, John Wiley, New York, 1983 
[24] E. Darrigrand, Coupling of fast multipole method and microlocal discretization for the 3-D Helmholtz equation, J. Comput. Phys., 181 (2002), pp. 126-154.

[25] E. Darve AND P. Havé, A fast multipole method for maxwell equations stable at all frequencies, Philos. Trans. R. Soc. London A, 362 (2004), pp. 603-628.

[26] A. De La Bourdonnaye, A microlocal discretization method and its utilization for a scattering problem, C.R. Acad. Sci. I Math., 318 (1994), pp. 385-388.

[27] A. De La Bourdonnaye and M. Tolentino, Reducing the condition number for microlocal discretization problems, Philos. Trans. R. Soc. London A, 362 (2004), pp. 541-559.

[28] M. Ganesh, S. Langdon, And I. H. Sloan, Efficient evaluation of highly oscillatory acoustic scattering surface integrals, Reading University Numerical Analysis Report 6/05, submitted for publication to J. Comp. Appl. Math.

[29] E. Giladi ANd J. Keller, A hybrid numerical asymptotic method for scattering problems, J. Comput. Phys., 174 (2001), pp. 226-247.

[30] D. Habault, Sound propagation above an inhomogeneous plane, J. Sound Vib., 100 (1985), pp. $55-67$.

[31] D. C. Hothersall and J. N. B. Harriott, Approximate models for sound propagation above multi-impedance plane boundaries, J. Acoust. Soc. Am., 97 (1995), pp. 918-926.

[32] T. Huttunen, P. Monk, F. Collino, and J. P. Kaipio, The ultra-weak variational formulation for elastic wave problems, SIAM J. Sci. Comput., 25 (2004), pp. 1717-1742.

[33] F. Ihlenburg, Finite Element Analysis of Acoustic Scattering, Springer-Verlag, New York, 1998.

[34] A. IsERLES, On the numerical quadrature of highly-oscillating integrals I: Fourier transforms, IMA J. Numer. Anal., 24 (2004), pp. 365-391.

[35] A. ISERLES, On the numerical quadrature of highly-oscillating integrals II: Irregular oscillations, IMA J. Numer. Anal., 25 (2005), pp. 25-44.

[36] I. M. Kaganova, The impedance boundary conditions and effective surface impedance of inhomogeneous metals, Phys. B Cond. Matter, 338 (2003), pp. 38-43.

[37] O. Laghrouche, P. Bettess, E. Perrey-Debain, and J. Trevelyan, Wave interpolation finite elements for helmholtz problems with jumps in the wave speed, Comput. Methods Appl. Mech. Engrg., 194 (2005), pp. 367-381.

[38] S. Langdon And S. N. Chandler-Wilde, A Galerkin boundary element method for an acoustic scattering problem, with convergence rate independent of frequency, in Proceedings of the 4th UK Conference on Boundary Integral Methods, S. Amini, ed., Salford University Press, 2003, pp. 67-76.

[39] S. Langdon and S. N. Chandler-Wilde, A GTD-based boundary element method for a surface scattering problem, Proc. Inst. Acoustics, 25 (2003), pp. 224-233.

[40] S. Langdon and I. G. GRaham, Boundary integral methods for singularly perturbed boundary value problems, IMA J. Numer. Anal., 21 (2001), pp. 217-237.

[41] P. Monk And D. Q. WANG, A least squares method for the Helmholtz equation, Comput. Methods Appl. Math., 175 (1999), pp. 121-136.

[42] P. M. Morse and K. U. Ingard, Theoretical Acoustics, McGraw-Hill, New York, 1968.

[43] E. Perrey-Debain, O. Lagrouche, P. Bettess, and J. Trevelyan, Plane-wave basis finite elements and boundary elements for three-dimensional wave scattering, Philos. Trans. R. Soc. London A, 362 (2004), pp. 561-577.

[44] E. Perrey-Debain, J. Trevelyan, and P. Bettess, Plane wave interpolation in direct collocation boundary element method for radiation and wave scattering: Numerical aspects and applications, J. Sound Vib., 261 (2003), pp. 839-858.

[45] E. Perrey-Debain, J. Trevelyan, and P. Bettess, Use of wave boundary elements for acoustic computations, J. Comput. Acoust., 11 (2003), pp. 305-321.

[46] E. Perrey-Debain, J. Trevelyan, And P. Bettess, On wave boundary elements for radiation and scattering problems with piecewise constant impedance, IEEE Trans. Ant. Prop., 53 (2005), pp. 876-879.

[47] J. R. Poirier, A. Bendali, And P. Borderies, Impedance boundary condition for rapidly oscillating surface scatterers, in Mathematical and Numerical Aspects of Wave Propagation, A. Bermudez, D. Gomez, P. Joly, and J. E. Roberts, eds., SIAM, Philadelphia, 2000, pp. 528-532.

[48] M. Shimoda, R. Iwaki, And M. Miyoshi, Scattering of an electromagnetic plane wave by a plane with local change of surface impedance, IEICE Trans. Electronics, E87C (2004), pp. $44-51$. 